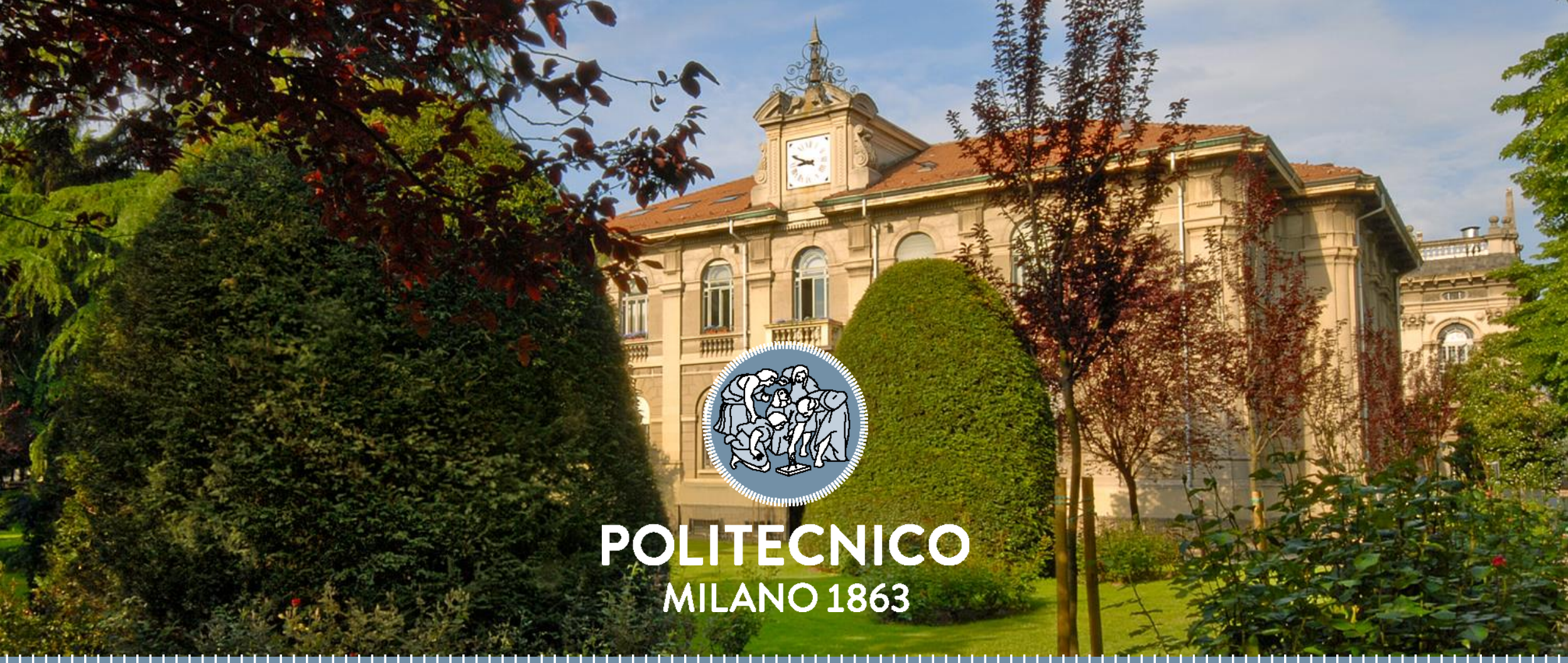

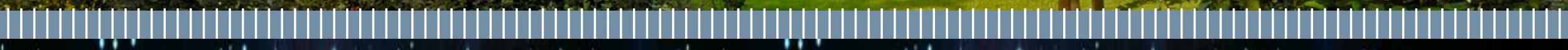
MCSI 2017 -4th International Conference on Mathematics and Co'mputers in Sciences and Industry - Córfu Island, Greece August 24-26,2017

The CICT IOU Reference Framework for Stronger AMS System Simulation in Science and Industry

Rodolfo A. Fiorini 
The CICT IOU Reference Framework for Stronger AMS System Simulation in Science and Industry

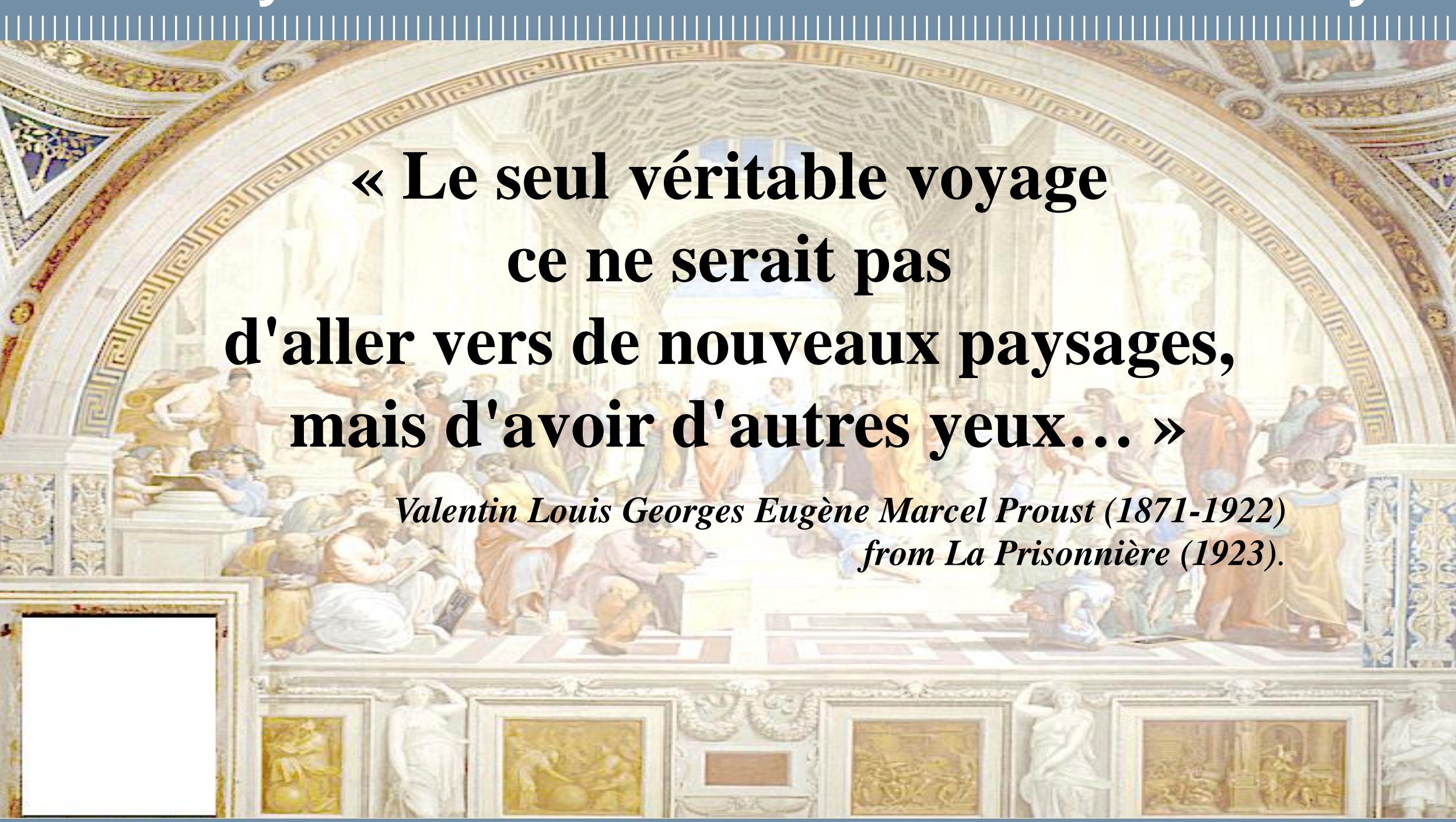

2 Rodolfo A. Fiorini 


\section{The CICT IOU Reference Framework for Stronger AMS System Simulation in Science and Industry}

\section{Presentation Outline}

1. Introduction (08)

- Robert Rosen Fundamental Modeling Relation

- Current Computational System Limitations

2. New Vision on Mathematics (05)

- CICT IOU Framework

- CICT Solution to the Problem for AMS System Modeling

3. New Predicative and Numerical Competence (17)

- Predicative Competence

- Numerical Competence

4. CICT Fundamental Concepts (20)

- CICT Discrete Riemannian Space

- Solid Number

5. Conclusion (06)

- Half-Plane Space vs. CICT OECS Space

- Current Landscape of Geometric Science of Information

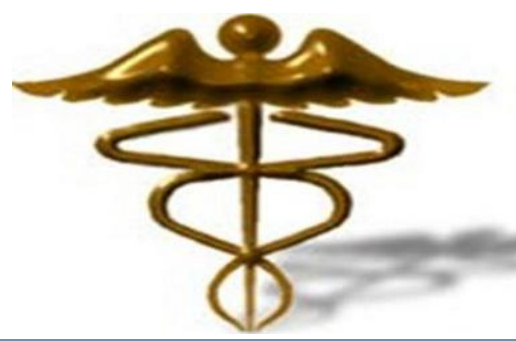




\section{The CICT IOU Reference Framework for Stronger AMS System Simulation in Science and Industry}

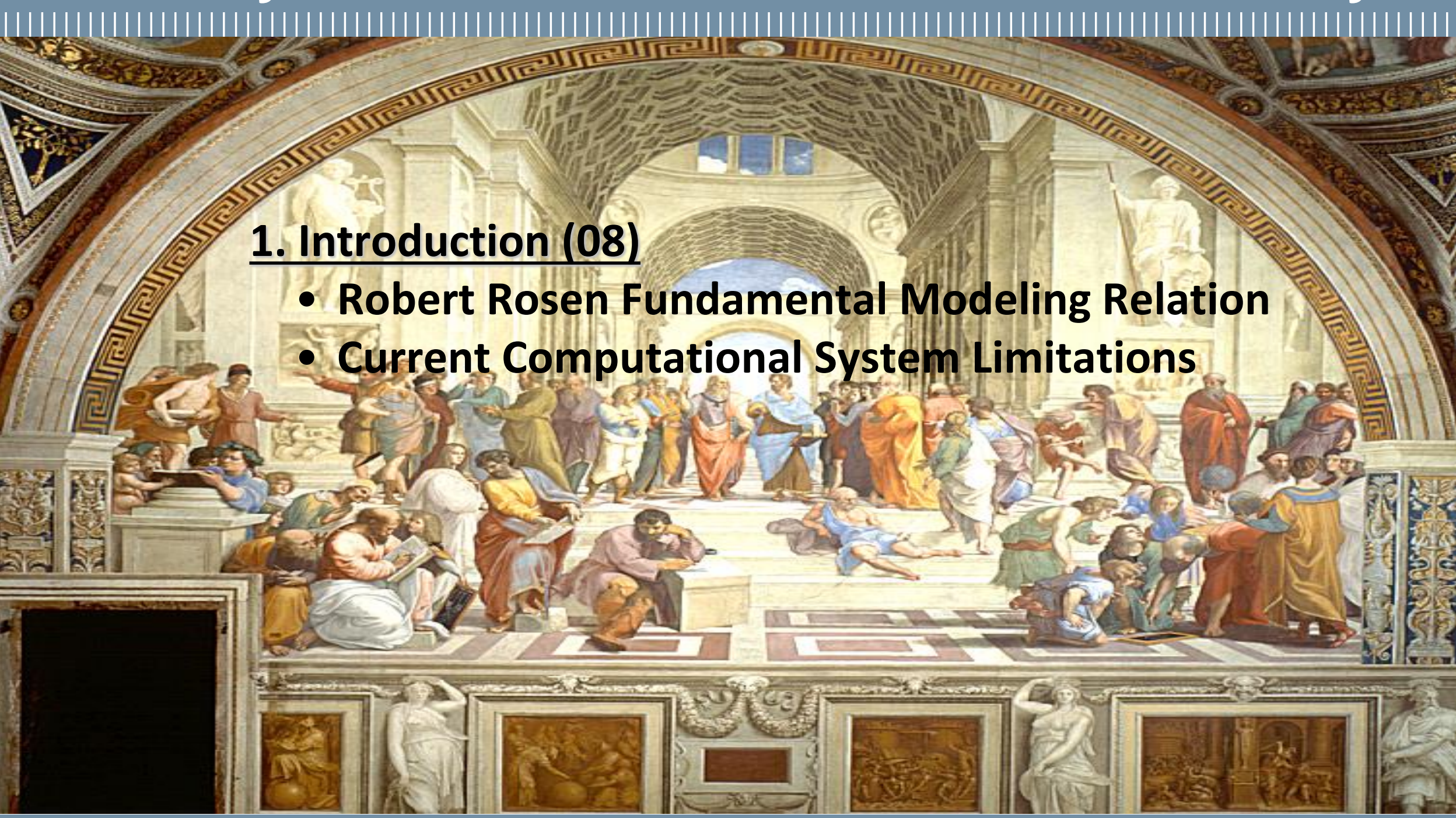

$4 \quad$ Rodolfo A. Fiorini

POLITECNICO MILANO 1863 


\section{The CICT IOU Reference Framework for Stronger AMS System Simulation in Science and Industry}

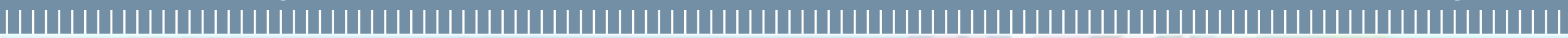

\section{Robert Rosen Fundamental Modeling Relation}

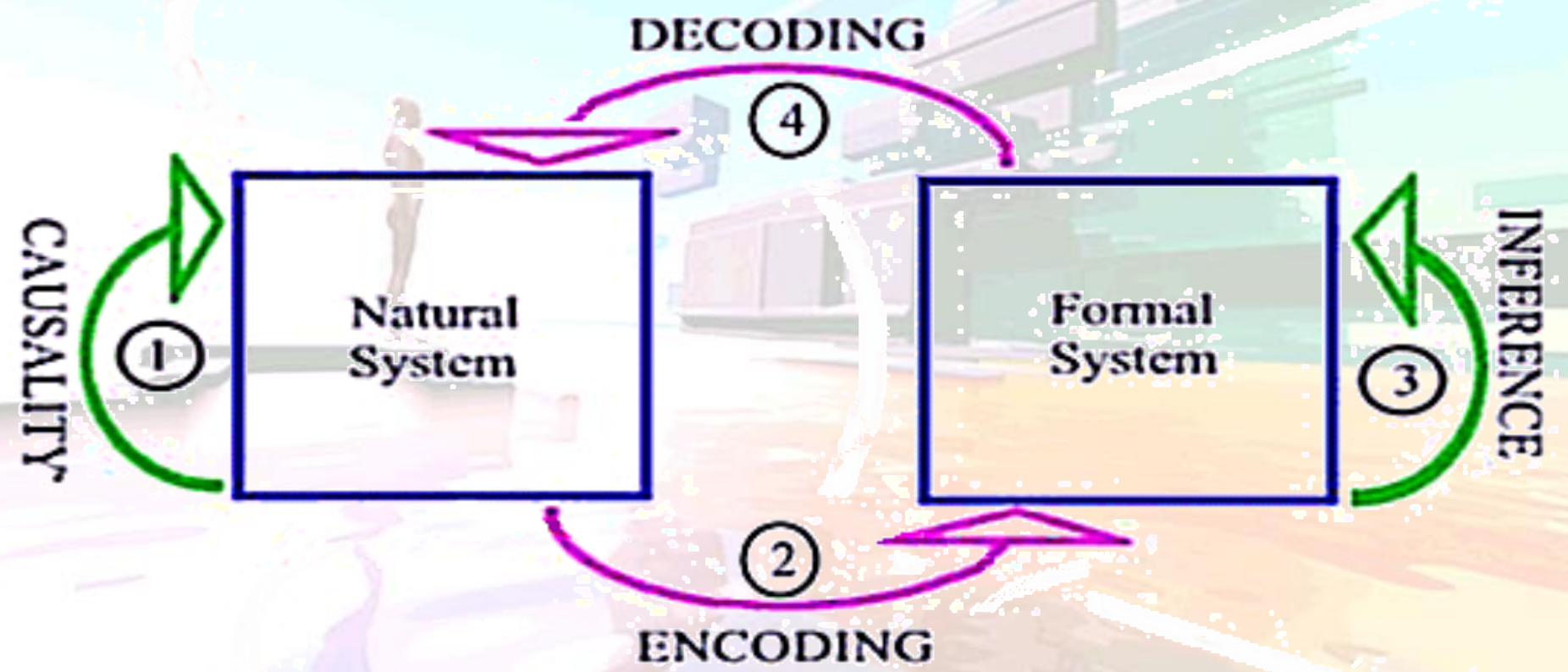

$5 \quad$ Rodolfo A. Fiorini 


\section{The CICT IOU Reference Framework for Stronger AMS System Simulation in Science and Industry}

The Root of the Current Problem for AMS System Modeling

\section{The Root of the Current Problem for AMS Syste

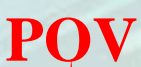

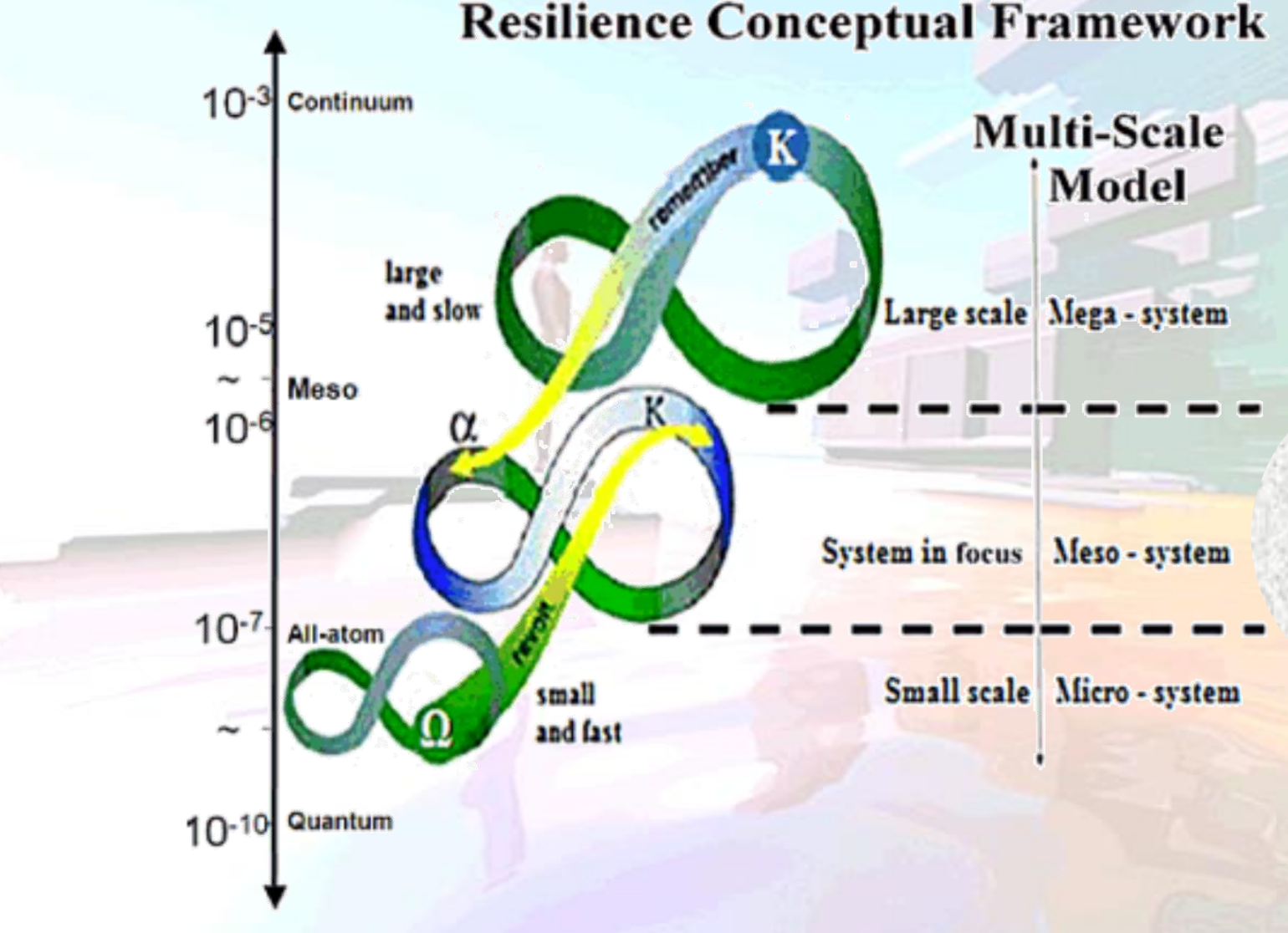

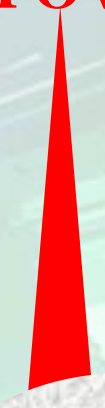




\section{The CICT IOU Reference Framework for Stronger AMS System Simulation in Science and Industry}

Current Number Theory and modern Numeric Analysis still use LTR (Left-To-Right) mono-directional interpretation only for numeric group generator and relations, so information entropy generation cannot be avoided in current computational algorithm and application.

Furthermore, traditional digital computational resources are unable to capture and to manage not only the full information content of a single Real Number $\boldsymbol{R}$, but even Rational Number $\boldsymbol{Q}$ is managed by information dissipation (e.g. finite precision machine, truncating, rounding, etc.).

So, paradoxically if you don't know the code used to communicate a message you can't tell the difference between an information-rich message and a random jumble of letters.

This is the information double-bind (IDB) problem in contemporary classic information theory and in current Science (nobody likes to talk about it). 


\section{The CICT IOU Reference Framework for Stronger AMS System Simulation in Science and Industry}

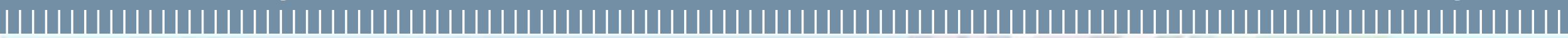

\section{Example: Image Lossless Compression Test}

( 4,096 by 4,096 pixel, $16,777,216$ true color image)

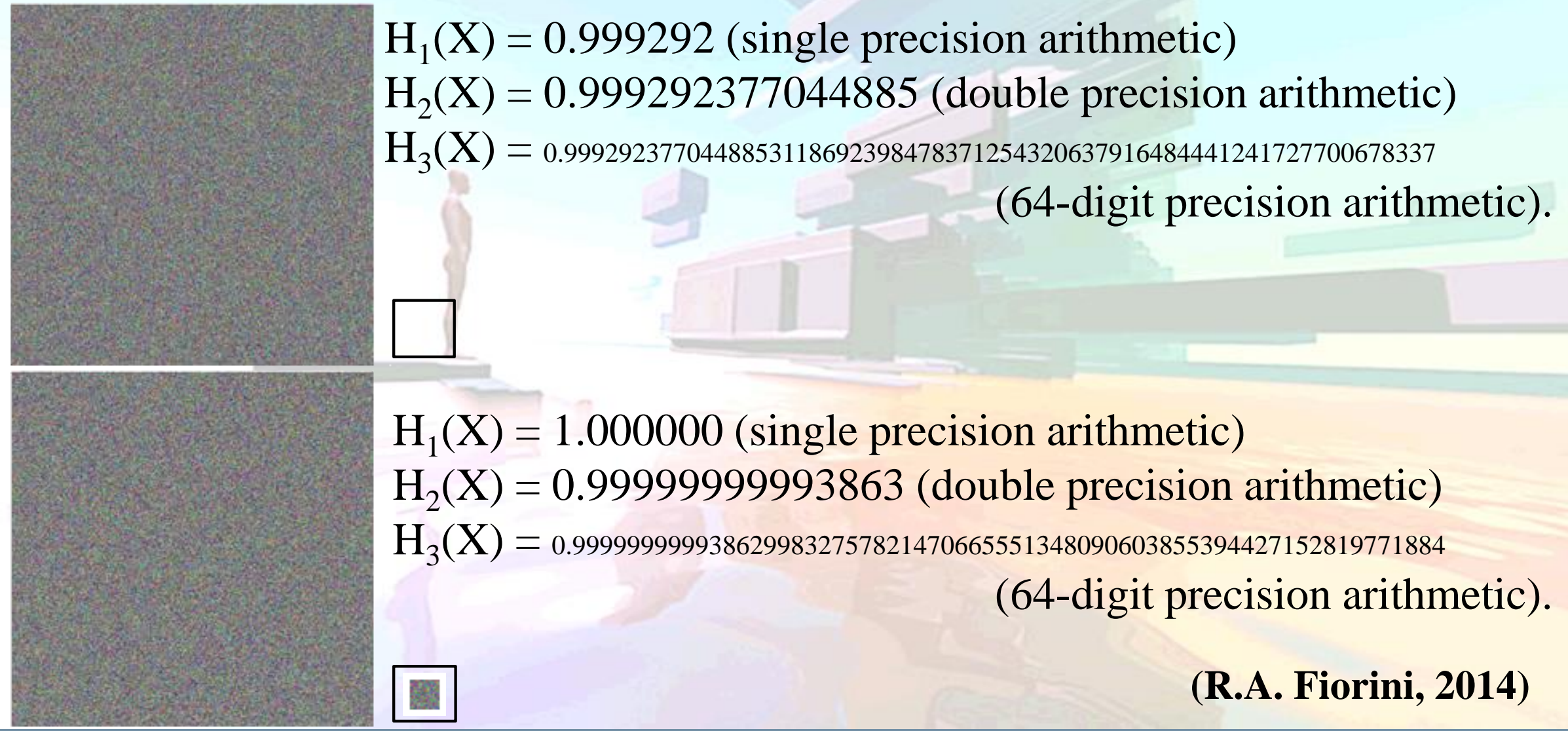

$8 \quad$ Rodolfo A. Fiorini 


\section{The CICT IOU Reference Framework for Stronger AMS System Simulation in Science and Industry}

\section{Current Computational System Limitations}

To achieve reliable system intelligence outstanding results, current computational system modeling and simulation community has to face and to solve two orders of modeling limitations at least, immediately:

(1) To develop stronger, more effective and reliable neural correlates by the correct arbitrary multi-scale (AMS) modeling approach for complex system;

(2) To minimize the traditional limitation of current digital computational resources that are unable to capture and to manage exactly even the full information content of a single Rational Number $\boldsymbol{Q}$, leading to computational noise, information dissipation and knowledge ambiguity and opacity.' 


\section{The CICT IOU Reference Framework for Stronger AMS System Simulation in Science and Industry}

\section{Computing with Numeric Words}

An intriguing point is that, although there are multiple models for the integers, they all will agree on the definition of computable functions. However, real number computation does not have these properties.

Scientific computation uses specified fixed-length finite representations (related to scientific notation) of real numbers, and so can achieve only limited precision, can make errors in comparisons, and can even be unstable over rounds of conversion to and from corresponding decimal representation.

Whether an extended Turing machine model or a real-number computation model is appropriate for scientific computation is still an open topic of discussion.

Current computer computation must be either symbolic or approximate.

Nevertheless it can be shown that computer computation can use either approximated approximation or exact approximation representation system. To achieve exact approximation computational number representation logic must be described in terms of closure spaces. 
The CICT IOU Reference Framework for Stronger AMS System Simulation in Science and Industry

Current Computational System Limitations

With no system coherent phase information, we get decoherence, entropy generation and information dissipation.

In fact, misplaced precision leads to information opacity, fuzziness, irreversibility, chaos, complexity and confusion. 
The CICT IOU Reference Framework for Stronger AMS System Simulation in Science and Industry

\section{Fundamental Question for New Vision}

\section{Is Classical Mathematics Appropriate for \\ Theory of Computation?}




\section{The CICT IOU Reference Framework for Stronger AMS System Simulation in Science and Industry}

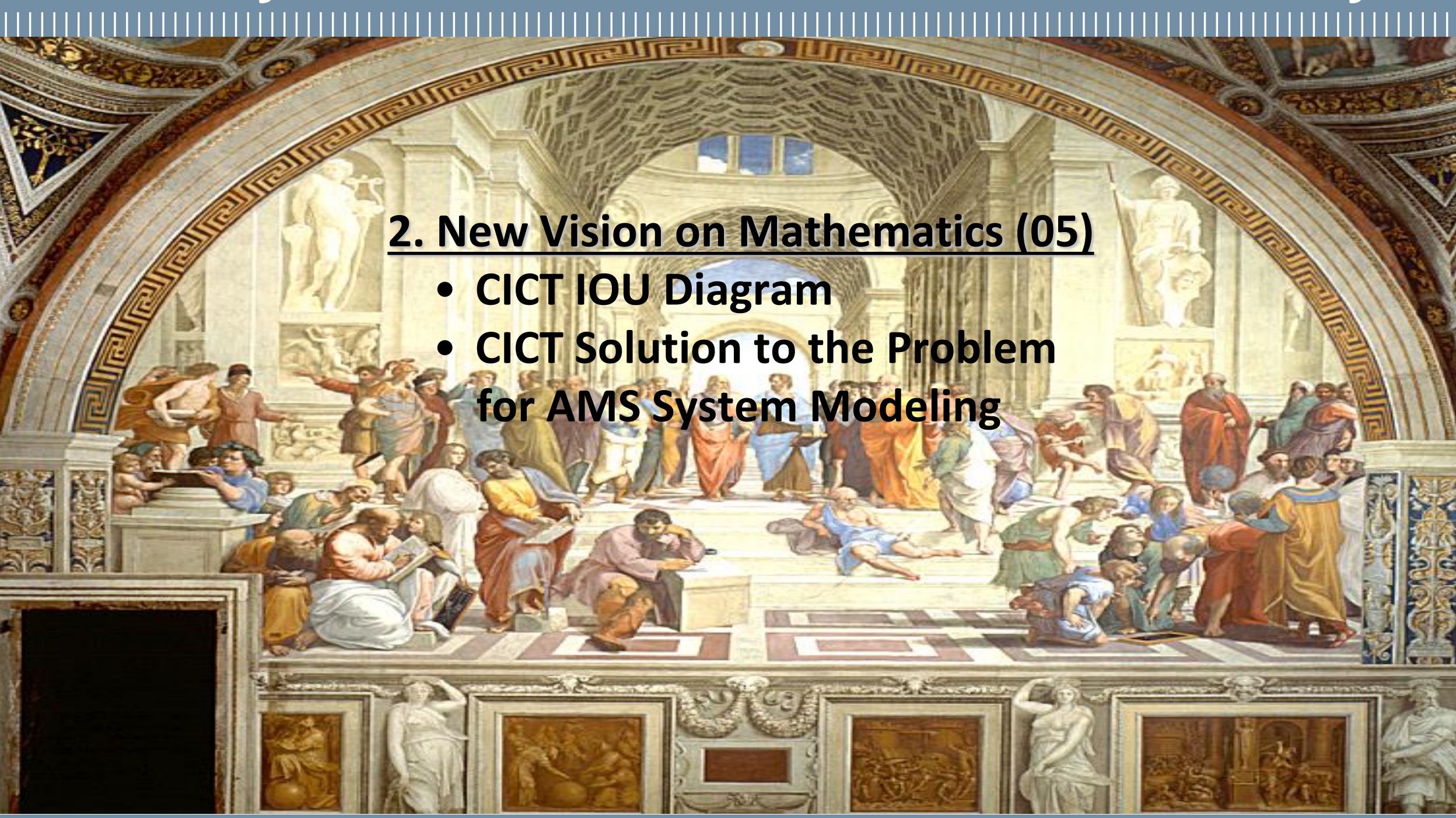

13 Rodolfo A. Fiorini

POLITECNICO MILANO 1863 


\section{The CICT IOU Reference Framework for Stronger AMS System Simulation in Science and Industry}

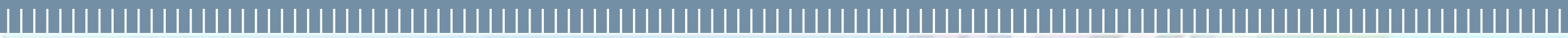
From Rosen Modeling Relation to ODR Modeling

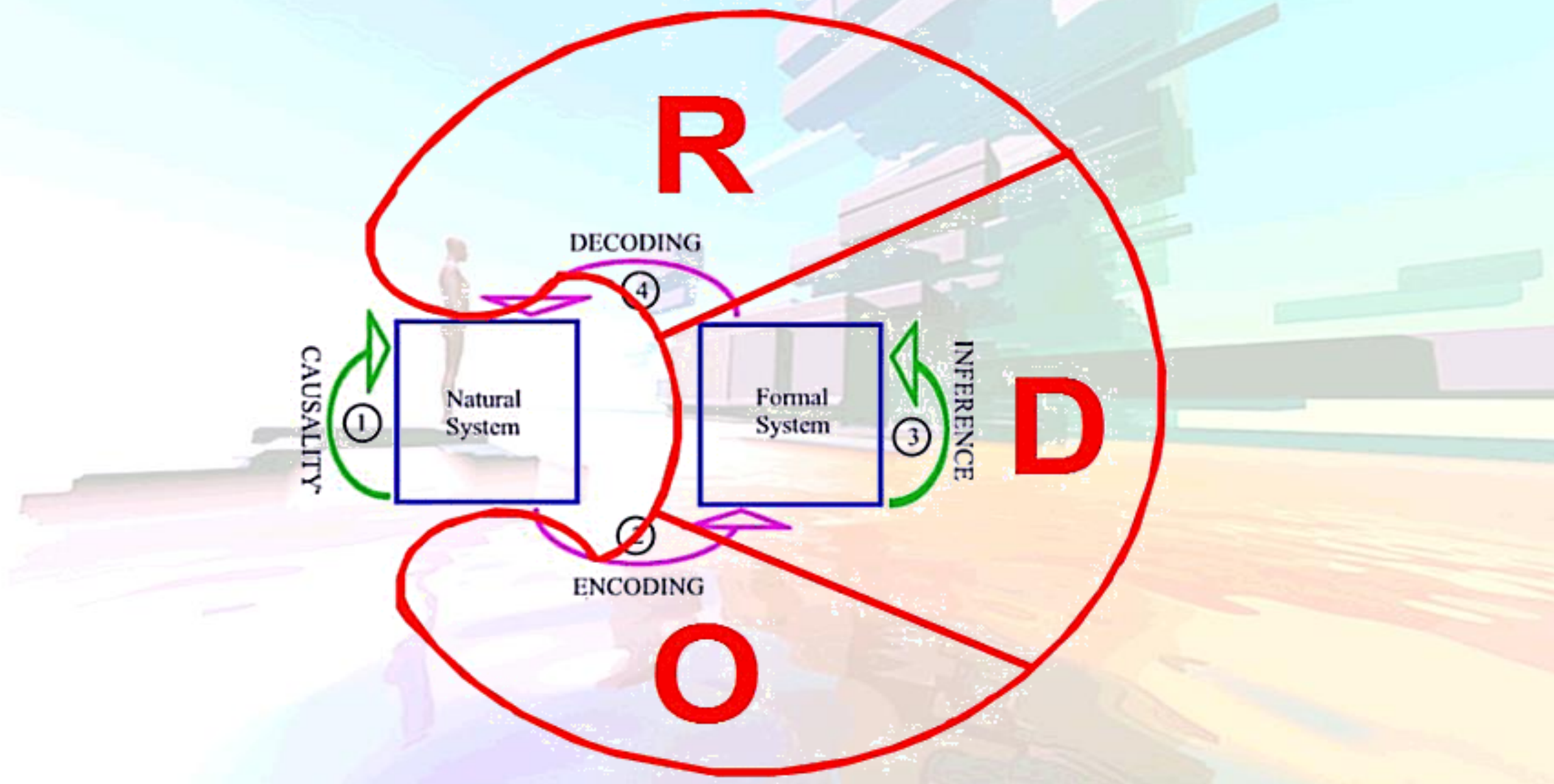




\section{The CICT IOU Reference Framework for Stronger AMS System Simulation in Science and Industry}

\section{CICT CO-ODR new awareness}

According to new "Computational Information Conservation Theory" (CICT) all computational information usually lost in the classic computational domain approach can be captured and recovered by corresponding ODR complementary co-domains, step-by-step. Then system co-domain information can be used to correct any computed result, achieving computational information conservation.

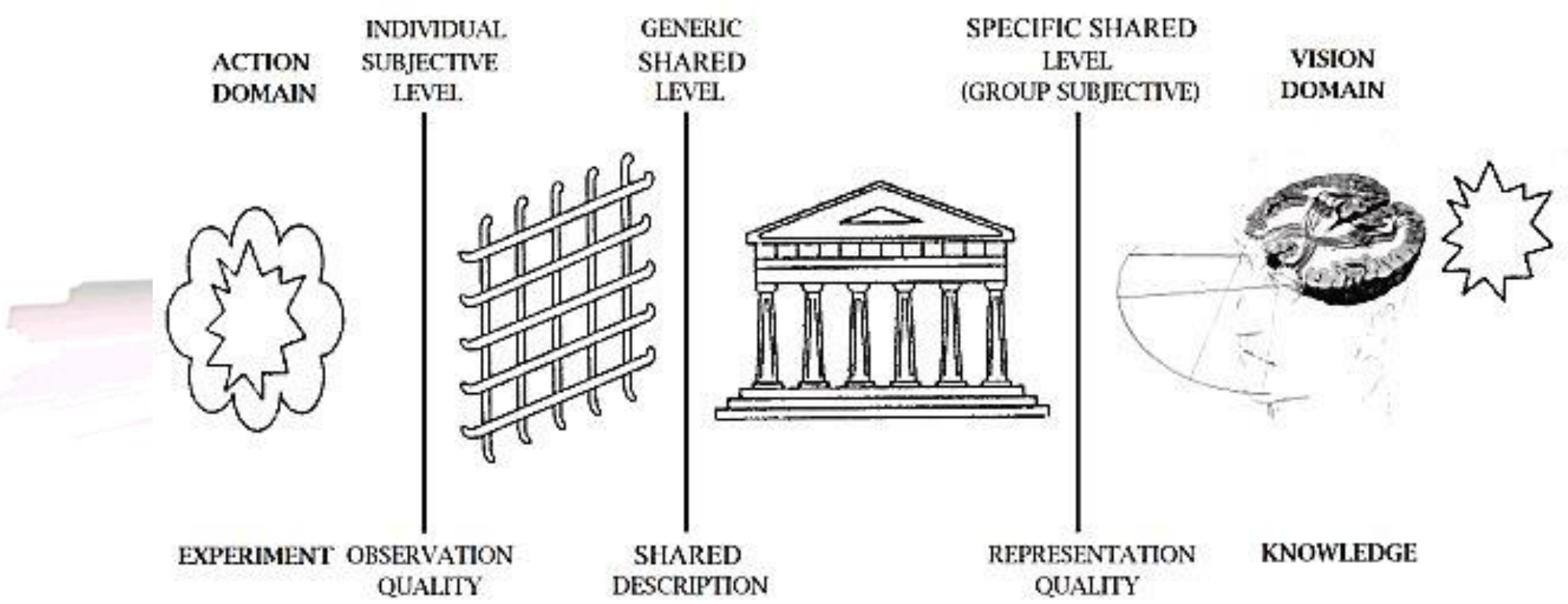




\section{The CICT IOU Reference Framework for Stronger AMS System Simulation in Science and Industry}

. CICT CO-ODR new awareness

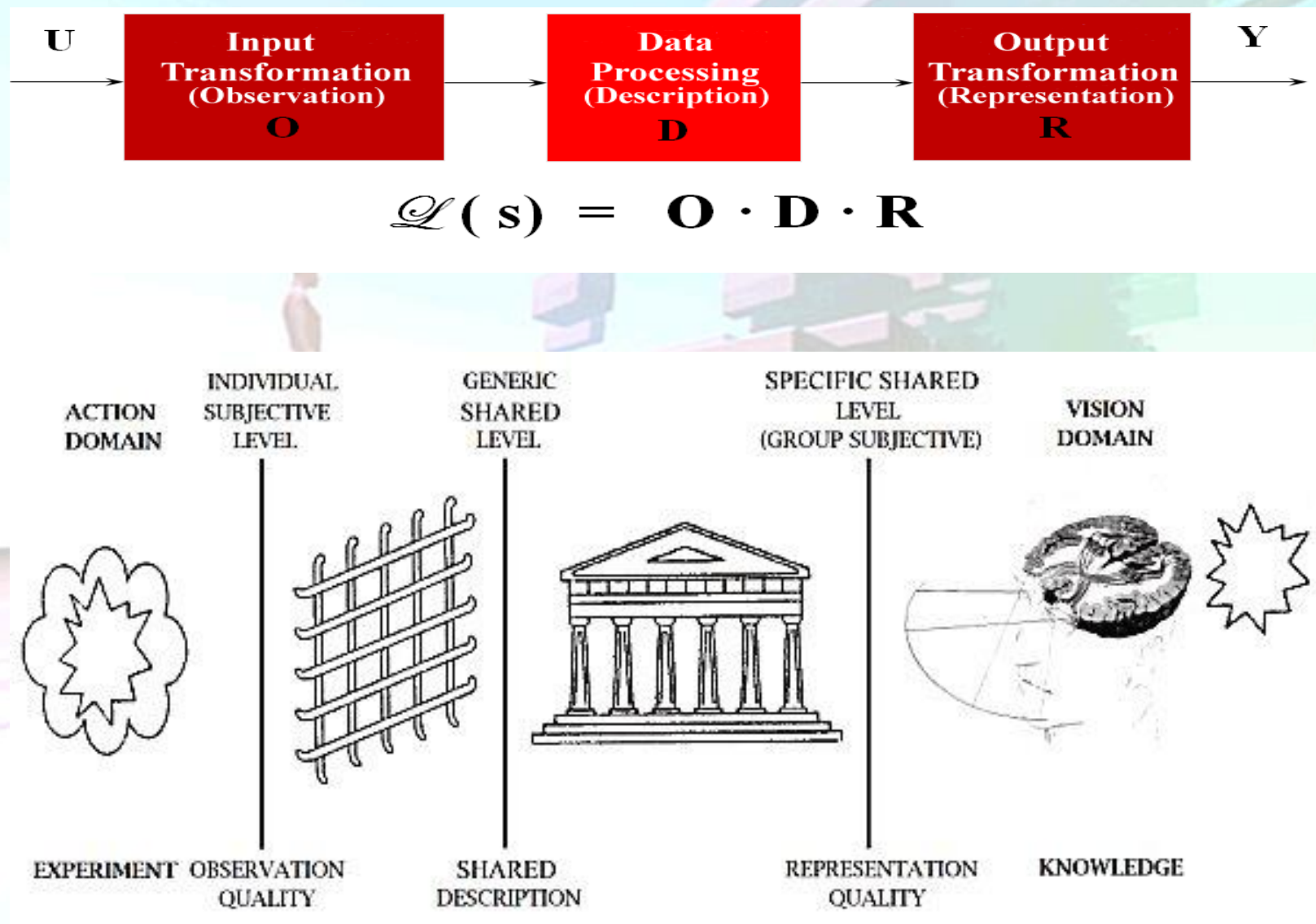


The CICT IOU Reference Framework for Stronger AMS System Simulation in Science and Industry

\section{CICT IOU Mapping By KLEIN Four-Group (CICT)}

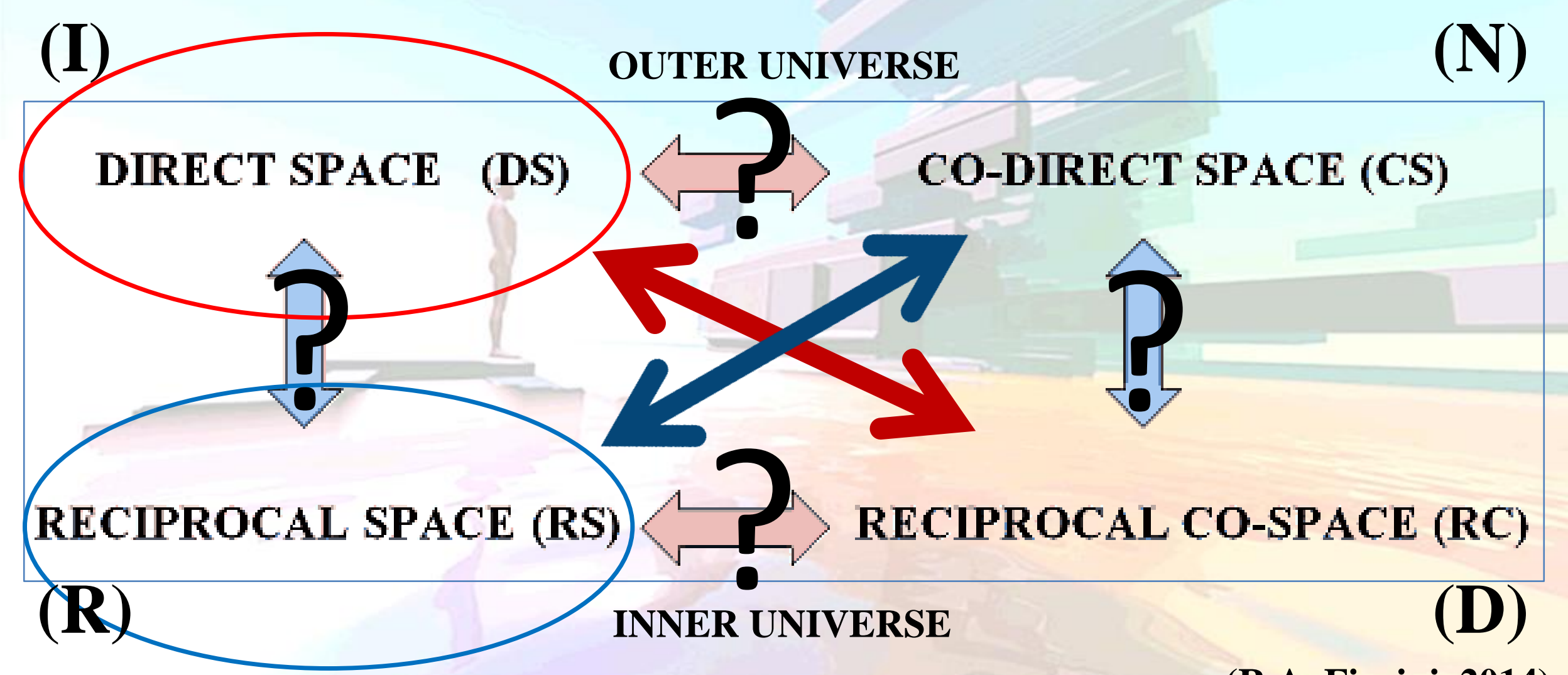

(R.A. Fiorini, 2014)

17 Rodolfo A. Fiorini 


\section{The CICT IOU Reference Framework for Stronger AMS System Simulation in Science and Industry}

CICT Solution to the Problem for AMS System Modeling

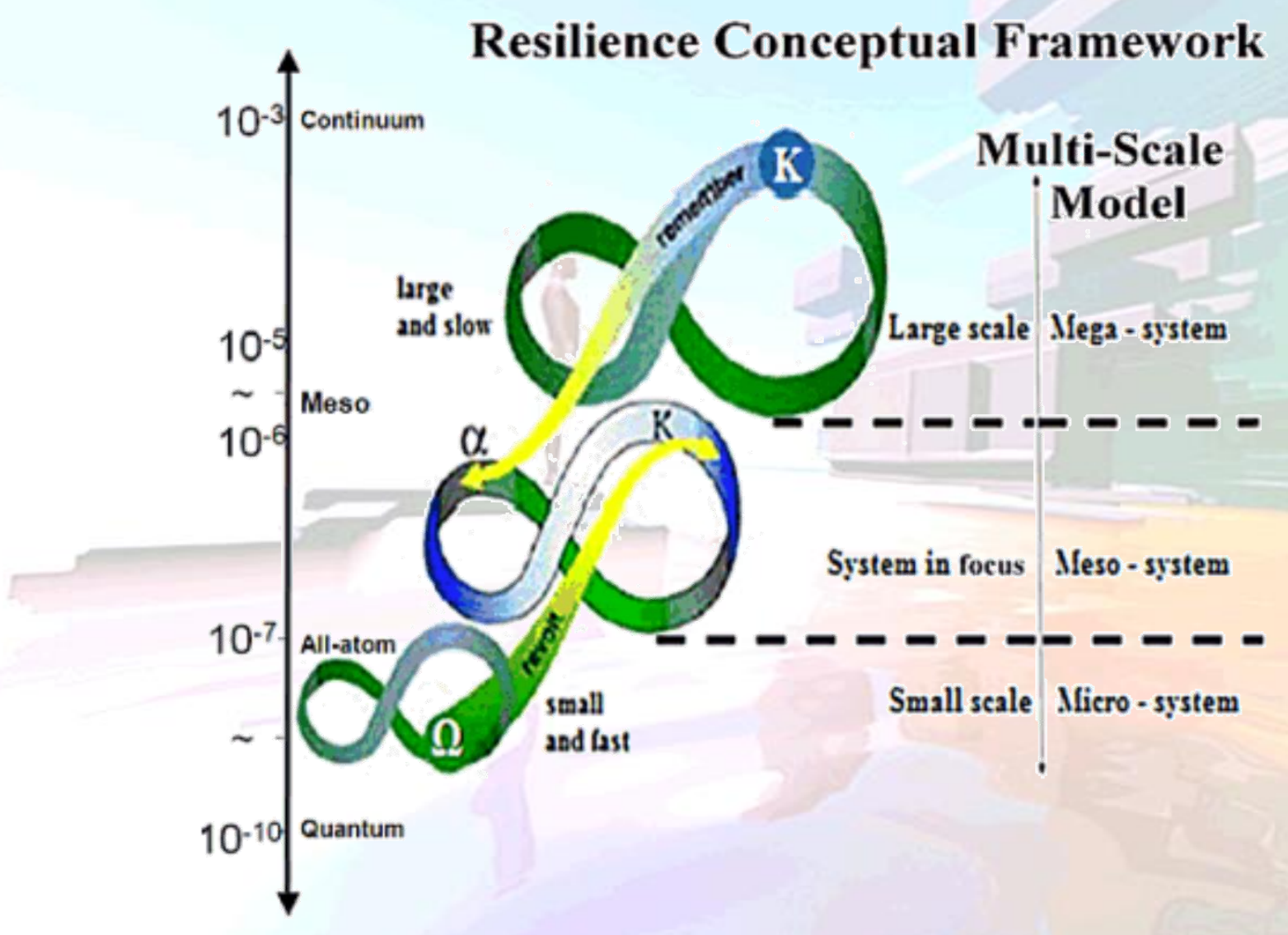

TD

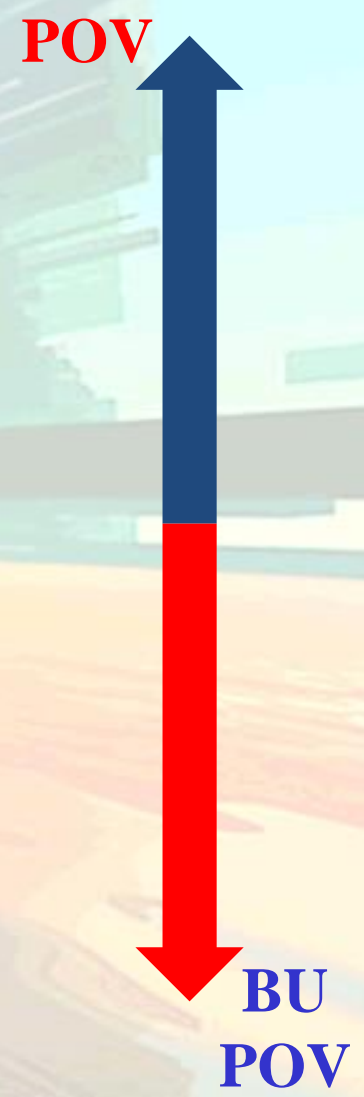

18 Rodolfo A. Fiorini 


\section{The CICT IOU Reference Framework for Stronger AMS System Simulation in Science and Industry}

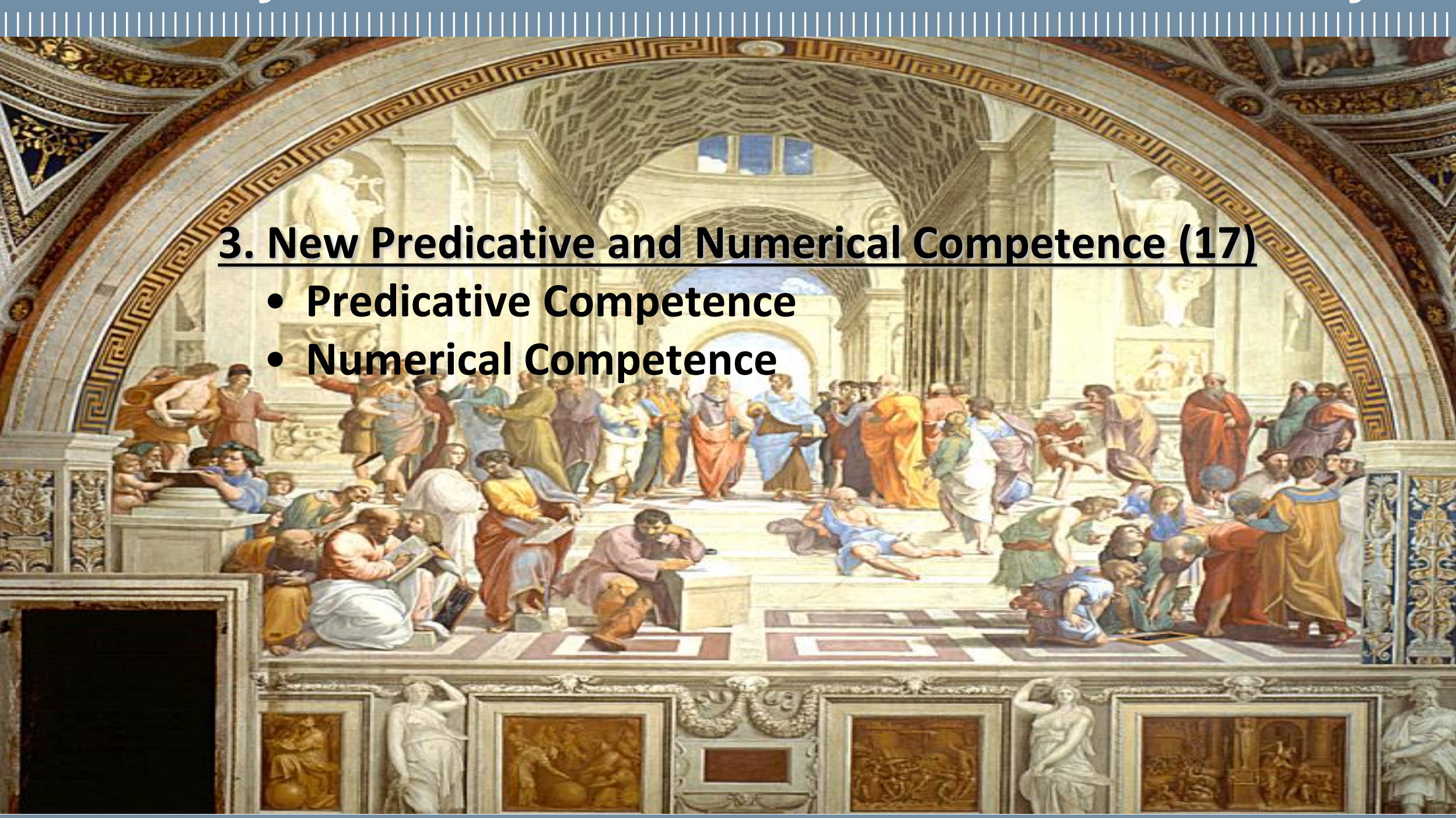

19 Rodolfo A. Fiorini

POLITECNICO MILANO 1863 


\section{The CICT IOU Reference Framework for Stronger AMS System Simulation in Science and Industry}
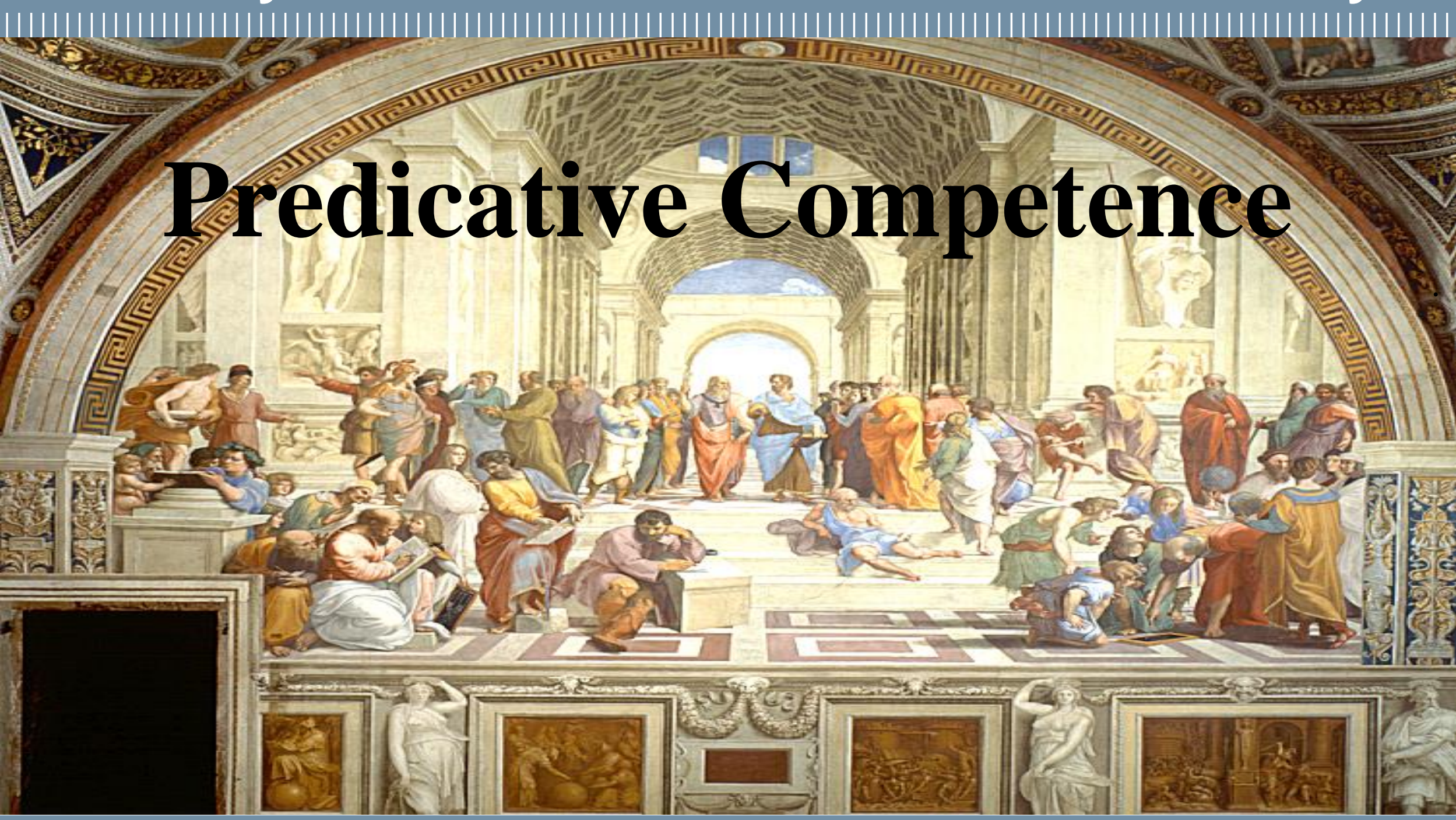

20 Rodolfo A. Fiorini

POLITECNICO MILANO 1863 


\section{The CICT IOU Reference Framework for Stronger AMS System Simulation in Science and Industry}

According to Swiss clinical psychologist Jean Piaget, human adults normally know how to use properly classical propositional logic. Piaget also held that the integration of algebraic composition and relational ordering in formal logic is realized via the mathematical Klein group structure (Inhelder and Piaget, 1955).

In the last fifty years, many experiments made by psychologists of reasoning have often shown most adults commit logical fallacies in propositional inferences. These experimental psychologists have so concluded, relying on many empirical evidences, that Piaget's claim about adults' competence in propositional logic was wrong and much too rationalist.

In other words, according to experimental psychologists, Piaget was overestimating the logical capacities of average human adults in the use of classical propositional logical connectives.

But, doing so, they forgot Piaget's rigorous and important analysis of the Klein group structure at work in logical competence. 
The CICT IOU Reference Framework for Stronger AMS System Simulation in Science and Industry

\section{Piaget's KLEIN Four-Group Definition}

\begin{tabular}{|l|l|l|l|l|}
\hline $\mathbf{x}$ & $\mathbf{I}$ & $\mathbf{N}$ & $\mathbf{R}$ & $\mathbf{D}$ \\
\hline $\mathbf{I}$ & $\mathbf{I}$ & $\mathrm{N}$ & $\mathrm{R}$ & $\mathrm{D}$ \\
\hline $\mathbf{N}$ & $\mathbf{N}$ & $\mathrm{I}$ & $\mathrm{D}$ & $\mathrm{R}$ \\
\hline $\mathbf{R}$ & $\mathrm{R}$ & $\mathrm{D}$ & $\mathrm{I}$ & $\mathbf{N}$ \\
\hline $\mathbf{D}$ & $\mathrm{D}$ & $\mathrm{R}$ & $\mathrm{N}$ & $\mathbf{I}$ \\
\hline
\end{tabular}

Inhelder and Piaget, 1955 
The CICT IOU Reference Framework for Stronger AMS System Simulation in Science and Industry

The Klein group structure generates Squares Of Opposition (SOOs), and an important component of human rationality resides in the diagram of the $\mathrm{SOO}$, as formal articulations of logical dependence between connectives (Beziau and Payette, 2012). 


\section{The CICT IOU Reference Framework for Stronger AMS System Simulation in Science and Industry}

\section{Aritotele's Syllogism: LOGIC}

\section{Basic Aristotele's "Square Of Oppositions“" (SOO)}

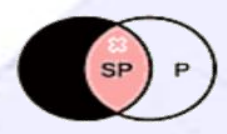

All $S$ are $P$.

SaP

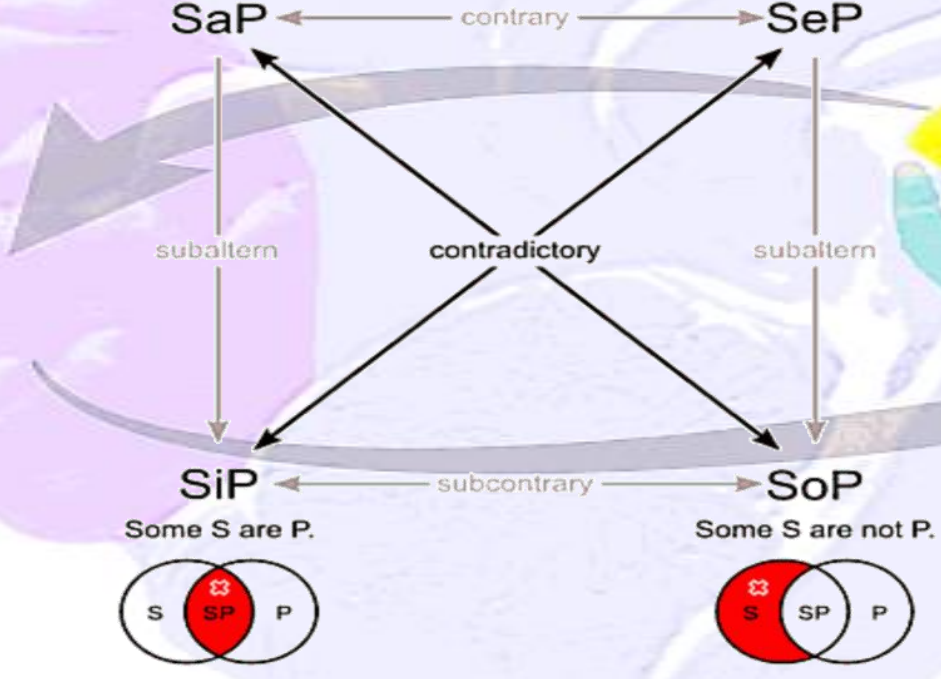

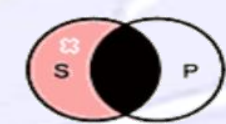

No $S$ is $P$

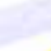

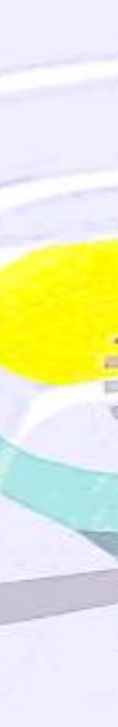
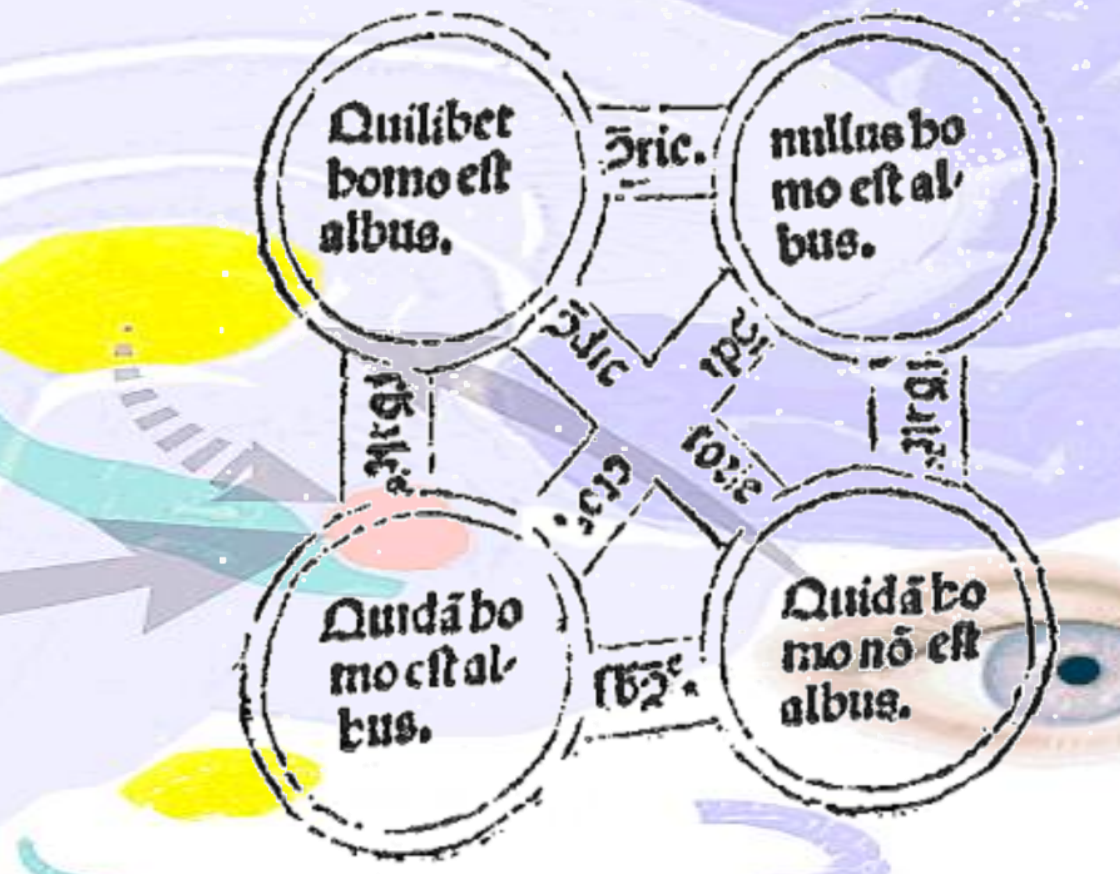

(Black areas are empty, red areas are nonempty) - Johannesmagistris-Square $\left(15^{\text {th }}\right.$ century)

(R.A. Fiorini, 2014) 


\section{The CICT IOU Reference Framework for Stronger AMS System Simulation in Science and Industry}

As a matter of fact, English talking people tend to treat conditionals as equivalences and inclusive disjunctions as being exclusive (Robert and Brisson, 2016).

We inevitably see the universe from a human point of view and communicate in terms shaped by the exigencies of human life in a natural uncertain environment. The diagram of the SOO is basic to formal articulations of logical dependence between connectives.

But the formal rationality provided by the SOO is not spontaneous and therefore, should not be easy to learn for adults.

This is the main reason why we need reliable and effective training tools to achieve full propositional logic proficiency in decision making, like the elementary pragmatic model (EPM) and the Evolutive EPM (E'PM) (De Giacomo and Fiorini, 2017). 


\section{The CICT IOU Reference Framework for Stronger AMS System Simulation in Science and Industry}

The Klein four-group is the smallest non-cyclic group, and every noncyclic group of order 4 is isomorphic to the Klein four-group.

The cyclic group of order $\mathbf{4}$ and the Klein four-group are therefore, up to isomorphism, the only groups of order 4 . Both are abelian groups in mathematics.

Piaget applied the Klein four-group to binary connectives, so that a given connective is associated first with itself (in an identical (I) transformation) and then with its algebraic complement (its inverse (N) transformation), also with its order opposite (its reciprocal (R) transformation) and finally, with the combination of its $\mathrm{N}$ and $\mathrm{R}$ transformations (that Piaget calls its "correlative" or C transformation) (Inhelder and Piaget,1955, ch.17.) This correlative corresponds to what logicians usually call the "dual" (D) transformation (Robert and Brisson, 2016).

The Klein group structure generates Squares Of Opposition (SOOs), and an important component of human rationality resides in the diagram of the SOO, as formal articulations of logical dependence between connectives (Beziau and Payette, 2012). 
The CICT IOU Reference Framework for Stronger AMS System Simulation in Science and Industry

\section{Klein Group Fundamental Properties}

Multiplicative Klein Group Cayley Table

Cyclic Group Cayley Table

\begin{tabular}{|c|c|c|c|c|}
\hline $\mathbf{X}$ & $\mathbf{e}$ & $\mathbf{a}$ & $\mathbf{b}$ & $\mathbf{c}$ \\
\hline $\mathbf{e}$ & $\mathrm{e}$ & $\mathrm{a}$ & $\mathrm{b}$ & $\mathrm{c}$ \\
\hline $\mathbf{a}$ & $\mathrm{a}$ & $\mathrm{e}$ & $\mathrm{c}$ & $\mathrm{b}$ \\
\hline $\mathbf{b}$ & $\mathrm{b}$ & $\mathrm{c}$ & $\mathrm{e}$ & $\mathrm{a}$ \\
\hline $\mathbf{c}$ & $\mathrm{c}$ & $\mathrm{b}$ & $\mathrm{a}$ & $\mathrm{e}$ \\
\hline
\end{tabular}

\begin{tabular}{|c|c|c|c|c|}
\hline $\mathbf{X}$ & $\mathbf{e}$ & $\mathbf{a}$ & $\mathbf{b}$ & $\mathbf{c}$ \\
\hline $\mathbf{e}$ & $\mathrm{e}$ & $\mathrm{a}$ & $\mathrm{b}$ & $\mathrm{c}$ \\
\hline $\mathbf{a}$ & $\mathrm{a}$ & $\mathrm{b}$ & $\mathrm{c}$ & $\mathrm{e}$ \\
\hline $\mathbf{b}$ & $\mathrm{b}$ & $\mathrm{c}$ & $\mathrm{e}$ & $\mathrm{a}$ \\
\hline $\mathbf{c}$ & $\mathrm{c}$ & $\mathrm{e}$ & $\mathrm{a}$ & $\mathrm{b}$ \\
\hline
\end{tabular}

27 Rodolfo A. Fiorini 


\section{The CICT IOU Reference Framework for Stronger AMS System Simulation in Science and Industry}
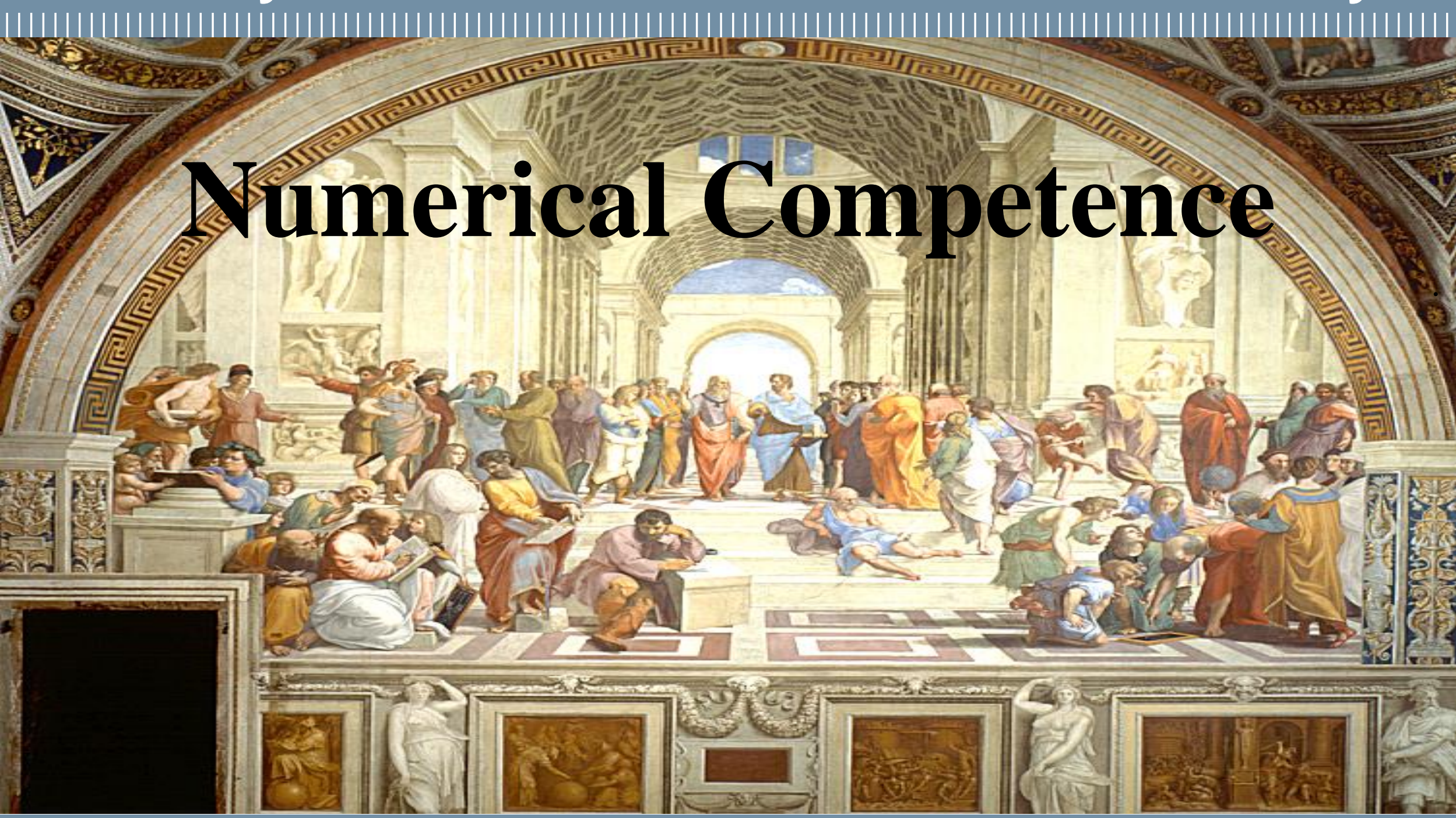

28 Rodolfo A. Fiorini

POLITECNICO MILANO 1863 


\section{The CICT IOU Reference Framework for Stronger AMS System Simulation in Science and Industry}

\section{CICT Computational Processing}

In CICT (Computational Information Conservation Theory) Arithmetic the structure of closure spaces is defined by Natural Numbers Reciprocal Space (RS) representation. By this way, Natural Number can be thought as both structured object and symbol.

Abstract structures do not represent objects but they are symbolic information representation only. They need an appropriate structural description first. Then we can formalize their semantics as a relationship between well-defined structures.

So we arrive to the fundamental difference in the ontological status of symbols and object represented by these symbols. To the difference that makes the difference.

CICT makes this fundamental ontological discrimination between Symbolic and OpeRational Number Representation to achieve computational information conservation. 


\section{The CICT IOU Reference Framework for Stronger AMS System Simulation in Science and Industry}

\section{New Vision on $Q$ Rational Number System}

CICT emerged from the study of the geometrical structure of a discrete manifold of ordered hyperbolic substructures, coded by formal power series, under the criterion of evolutive structural invariance at arbitrary precision.

It defines an arbitrary-scaling discrete Riemannian manifold uniquely, under HG metric, that, for arbitrary finite point accuracy level $\boldsymbol{L}$ going to infinity (exact solution theoretically), is homeomorphic to traditional Information Geometry Riemannian manifold.

In other words, HG can describe a projective relativistic geometry directly hardwired into elementary arithmetic long division remainder sequences, offering many competitive computational advantages over traditional Euclidean approach. 


\section{The CICT IOU Reference Framework for Stronger AMS System Simulation in Science and Industry}

\section{New Vision on $Q$ Rational Number System}

CICT emerged from the study of the geometrical structure of a discrete manifold of ordered hyperbolic substructures, coded by formal power series, under the criterion of evolutive structural invariance at arbitrary precision.

It defines an arbitrary-scaling discrete Riemannian manifold uniquely, under HG metric, that, for arbitrary finite point accuracy level $\boldsymbol{L}$ going to infinity (exact solution theoretically), is homeomorphic to traditional Information Geometry Riemannian manifold.

In other words, HG can describe a projective relativistic geometry directly hardwired into elementary arithmetic long division remainder sequences, offering many competitive computational advantages over traditional Euclidean approach. 


\section{The CICT IOU Reference Framework for Stronger AMS System Simulation in Science and Industry}

\section{New Vision on $Q$ Rational Number System}

Elementary Arithmetic long Division minority components (Remainders, $\mathrm{R}$ ), for long time, concealed relational knowledge to their dominant result (Quotient, Q), not only can always allow quotient regeneration from their remainder information to any arbitrary precision, but even to achieve information conservation and coding minimization, by combinatorial OECS (Optimized Exponential Cyclic Sequences), for dynamical systems.

Then traditional $\boldsymbol{Q}$ Arithmetic can be even regarded as a highly sophisticated open logic, powerful and flexible LTR and RTL formal numeric language of languages, with self-defining consistent word and rule, starting from elementary generator and relation.

This new awareness can guide the development of successful more convenient algorithm, application and powerful computational system.

(Fiorini \& Laguteta, 2013) 


\section{The CICT IOU Reference Framework for Stronger AMS System Simulation in Science and Industry}

The CICT fundamental relationship that ties together numeric body information of divergent and convergent monotonic power series in any base (in this case decimal, with no loss of generality), with $\boldsymbol{D}$ ending by digit $\mathbf{9}$, is given by the following CICT fundamental LTR-RTL correspondence equation:

$$
\frac{1}{D}=\sum_{k=0}^{\infty} \frac{1}{10^{W}}\left(\frac{\bar{D}}{10^{W}}\right)^{k} \Rightarrow \ldots \Leftarrow \operatorname{Div}\left(\frac{1}{D}\right)=\sum_{k=0}^{\infty}(D+1)^{k}
$$

where $\bar{D}$ is the additive $10^{\mathrm{W}}$ complement of $D$, i.e. $\bar{D}=\left(10^{\mathrm{W}}-D\right)$,

$\mathrm{W}$ is the word representation precision length of the denominator $D$ and "Div" means "Divergence of".

Further generalizations related to $D$ ending by digit 1 or 3 or 7 are straightforward.

Increasing the level of representation accuracy, the total number of allowed convergent paths to $1 / D$, as allowed conservative paths, increases accordingly and can be counted exactly, till maximum machine word length and beyond: like discrete quantum paths denser and denser to one another, towards a never ending "blending quantum continuum," by a TD system perspective.

(Fiorini \& Laguteta, 2013) 


\section{The CICT IOU Reference Framework for Stronger AMS System Simulation in Science and Industry}

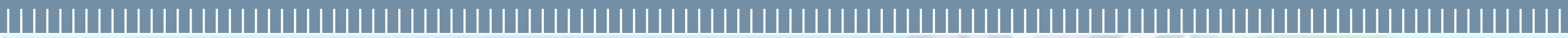
Domain of z-transforms

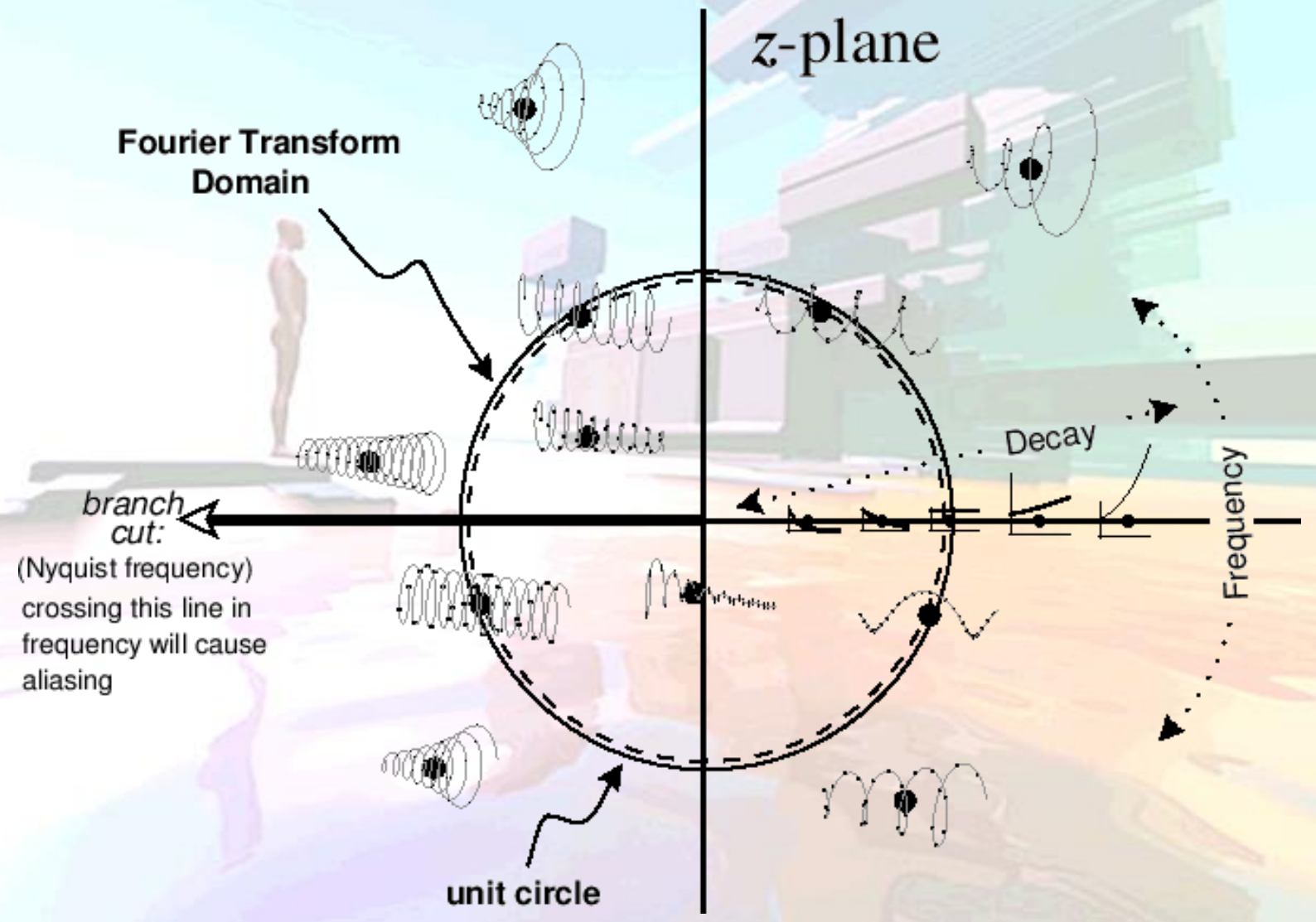




\section{The CICT IOU Reference Framework for Stronger AMS System Simulation in Science and Industry}

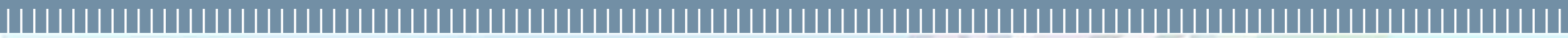

\section{Domain of Laplace transforms}

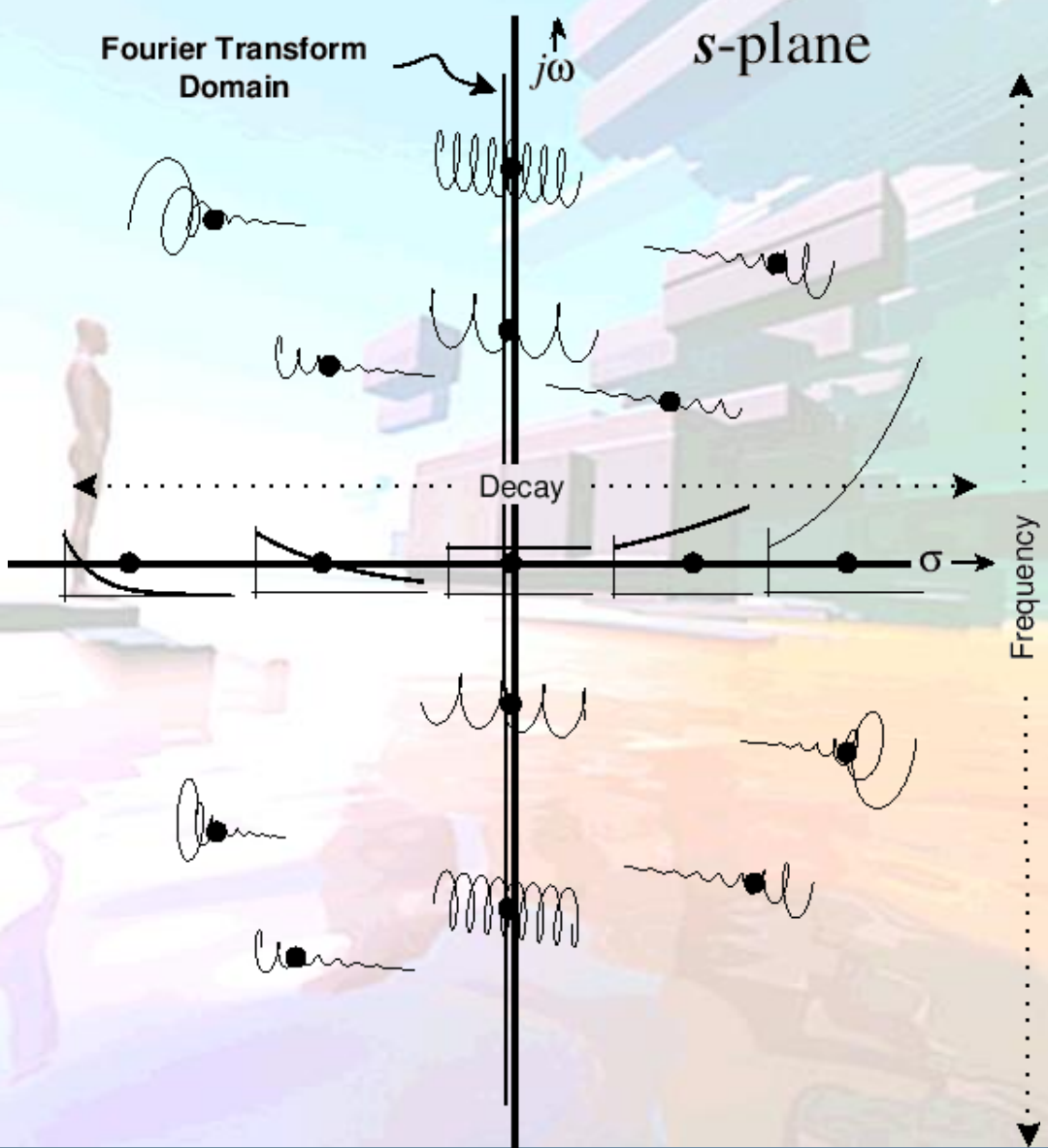

35 Rodolfo A. Fiorini 


\section{The CICT IOU Reference Framework for Stronger AMS System Simulation in Science and Industry}

Current Classic Information Processing Duality

Is Based on Two Coupled Irreducible Complementary Subsystems

\section{Linguistic Processing}

\section{Computational Processing}




\section{The CICT IOU Reference Framework for Stronger AMS System Simulation in Science and Industry}

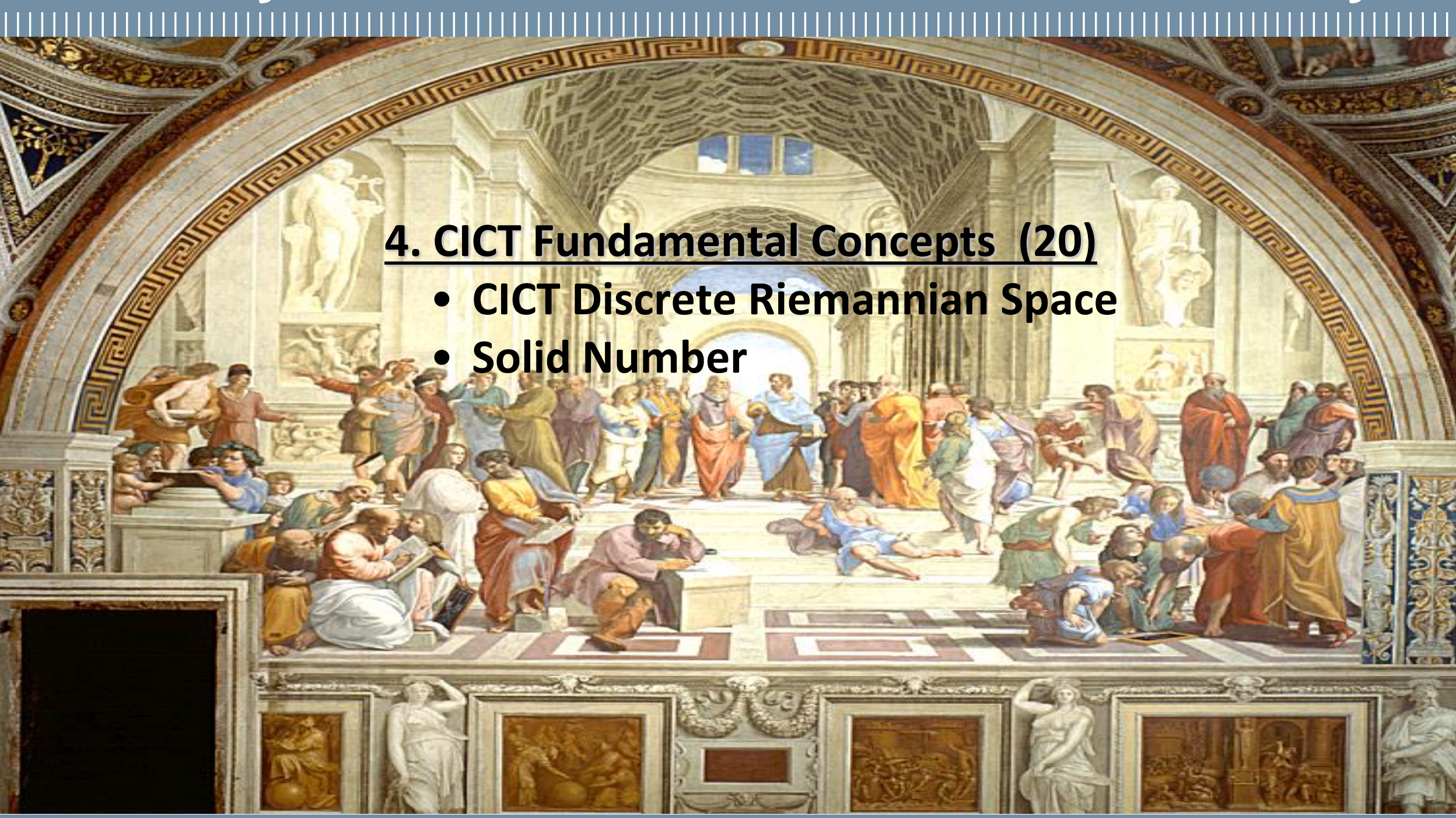

37 Rodolfo A. Fiorini

POLITECNICO MILANO 1863 
The CICT IOU Reference Framework for Stronger AMS System Simulation in Science and Industry

ишишиш System Simulation in Science and Industry

CICT Main Presentation Systems

CICT

Formal Power Series (FPS)

Presentation

vS.

CICT

Formal Recurrence Sequence (FRS) Presentation 


\section{The CICT IOU Reference Framework for Stronger AMS System Simulation in Science and Industry}

\section{New Vision on Rational Number System D $=49$}

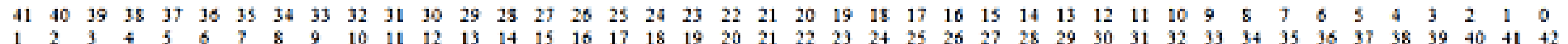

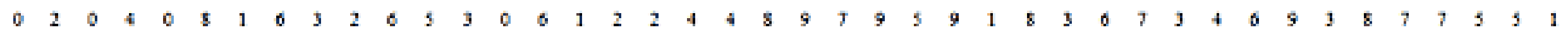

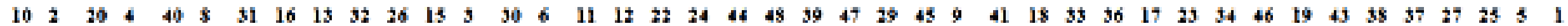

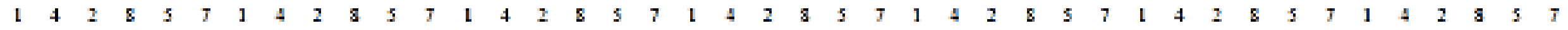

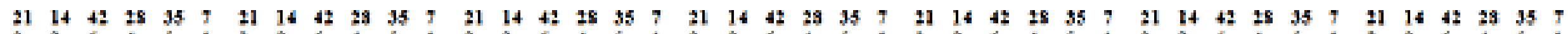

Formal Recurrence Sequence (FRS)

\section{Presentation}

$Q \equiv a_{n}=5 a_{n-1}+1 a_{n-2}, \quad a_{0}=0, a_{1}=1$

$R \equiv a_{n}=5 a_{n-1}+1 a_{\mathrm{H}-2} \quad \bmod (49), \quad a_{0}=10, a_{1}=2$

\section{Formal Power Series (FPS)}

\section{Presentation}

$$
\begin{aligned}
& Q \equiv \sum_{k=0}^{\infty} \frac{1}{100}\left(\frac{51}{100}\right)^{k}=\sum_{k=0}^{\infty} \frac{2}{100}\left(\frac{2}{100}\right)^{k} \\
& R \equiv 10^{k} \bmod (49) \quad k=1,2,3, \ldots
\end{aligned}
$$




\section{The CICT IOU Reference Framework for Stronger AMS System Simulation in Science and Industry}

\section{Solid Number (SN = 7) Family Group (first order)} OECS Modular Trajectory Rescaling

$$
\left(\text { Precision }=10^{-2}\right. \text { ) }
$$

Geometric Series Representation Compact Representation

$$
\begin{gathered}
\frac{1}{07}=\sum_{k=0}^{\infty} \frac{1}{10^{2}}\left(\frac{93}{10^{2}}\right)^{k}, \\
\frac{1}{07}=\sum_{k=0}^{\infty} \frac{2}{10^{2}}\left(\frac{86}{10^{2}}\right)^{k}, \\
\frac{1}{07}=\sum_{k=0}^{\infty} \frac{3}{10^{2}}\left(\frac{79}{10^{2}}\right)^{k}, \\
\vdots \\
\frac{1}{07}=\sum_{k=0}^{\infty} \frac{14}{10^{2}}\left(\frac{02}{10^{2}}\right)^{k} .
\end{gathered}
$$

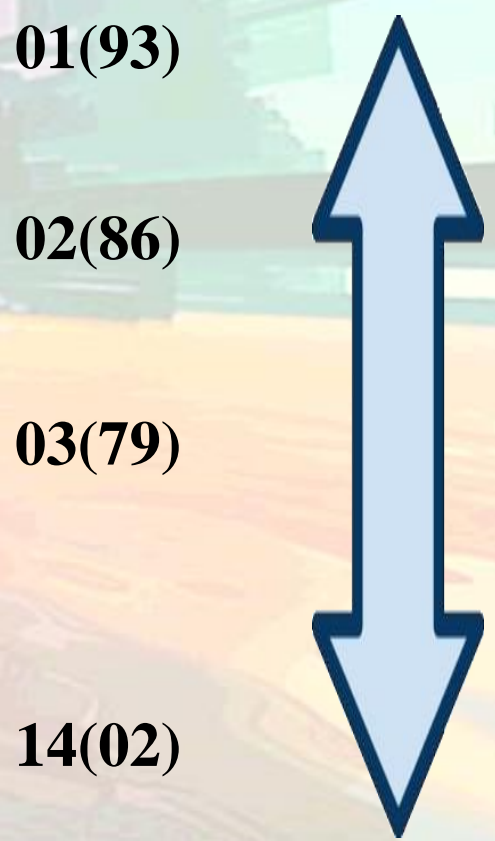

40 Rodolfo A. Fiorini 


\section{The CICT IOU Reference Framework for Stronger AMS System Simulation in Science and Industry}

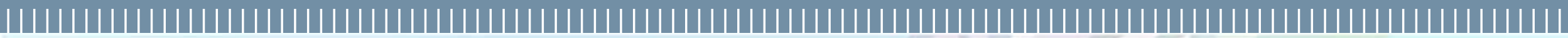

\section{OECS Word Space Example $\left(\right.$ Precision $\left.=10^{-2}\right)$}

\begin{tabular}{|c|c|c|c|c|c|c|c|c|c|c|c|c|c|c|c|c|c|}
\hline 1/ & $1 /$ & $1 /$ & 1) & 1/ & 1) & $1 /$ & 1/ & 1) & $1 /$ & $1 /$ & $1 /$ & 1f & $1 /$ & $1 /$ & $1 /$ & 1/ & $1 /$ \\
\hline 03 & 04 & 05 & 06 & 07 & 08 & 09 & 10 & 11 & 12 & 13 & 14 & 15 & 16 & 17 & 18 & 19 & 20 \\
\hline $\begin{array}{l}37 \\
\text { (11) }\end{array}$ & $\begin{array}{l}29 \\
\text { (16) }\end{array}$ & $\begin{array}{l}24 \\
(20)\end{array}$ & $\begin{array}{l}20 \\
(20)\end{array}$ & $\begin{array}{l}18 \\
(26)\end{array}$ & $\begin{array}{l}16 \\
(28)\end{array}$ & $\begin{array}{l}15 \\
(35)\end{array}$ & $\begin{array}{l}14 \\
(40)\end{array}$ & $\begin{array}{l}13 \\
\text { (43) }\end{array}$ & $\begin{array}{l}12 \\
(44)\end{array}$ & $\begin{array}{l}11 \\
(43)\end{array}$ & $\frac{11}{(54)}$ & $\begin{array}{l}10 \\
(50)\end{array}$ & $\begin{array}{l}10 \\
(60)\end{array}$ & $\begin{array}{l}09 \\
\text { (53) }\end{array}$ & $\begin{array}{l}09 \\
(62)\end{array}$ & $\begin{array}{l}09 \\
\text { (71) }\end{array}$ & $\begin{array}{l}09 \\
(80)\end{array}$ \\
\hline $\begin{array}{l}36 \\
(08)\end{array}$ & $\begin{array}{l}28 \\
(12)\end{array}$ & $\begin{array}{l}23 \\
(15)\end{array}$ & $\begin{array}{l}19 \\
(14)\end{array}$ & $\begin{array}{l}17 \\
(19)\end{array}$ & $\begin{array}{l}15 \\
(20)\end{array}$ & $\begin{array}{l}14 \\
(26)\end{array}$ & $\begin{array}{l}13 \\
(30)\end{array}$ & $\begin{array}{l}12 \\
(32)\end{array}$ & $\begin{array}{l}11 \\
(32)\end{array}$ & $\begin{array}{l}10 \\
(30)\end{array}$ & $\frac{10}{(40)}$ & $\begin{array}{l}09 \\
(35)\end{array}$ & $\begin{array}{l}09 \\
(44)\end{array}$ & $\begin{array}{l}08 \\
(36)\end{array}$ & $\begin{array}{l}08 \\
(44)\end{array}$ & $\begin{array}{l}08 \\
(52)\end{array}$ & $\begin{array}{l}08 \\
(60)\end{array}$ \\
\hline $\begin{array}{l}35 \\
(05)\end{array}$ & $\begin{array}{l}27 \\
(08)\end{array}$ & $\begin{array}{l}22 \\
(10)\end{array}$ & $\begin{array}{l}18 \\
(08)\end{array}$ & $\begin{array}{l}16 \\
(12)\end{array}$ & $\begin{array}{l}14 \\
(12)\end{array}$ & $\begin{array}{l}13 \\
\text { (17) }\end{array}$ & $\begin{array}{l}12 \\
(20)\end{array}$ & $\begin{array}{l}11 \\
(21)\end{array}$ & $\begin{array}{l}10 \\
(20)\end{array}$ & $\begin{array}{l}09 \\
(17)\end{array}$ & $\begin{array}{l}09 \\
(26)\end{array}$ & $\begin{array}{l}08 \\
(20)\end{array}$ & $\begin{array}{l}08 \\
(28)\end{array}$ & $\begin{array}{l}07 \\
\text { (19) }\end{array}$ & $\begin{array}{l}07 \\
(26)\end{array}$ & $\begin{array}{l}07 \\
\text { (33) }\end{array}$ & $\begin{array}{l}07 \\
(40)\end{array}$ \\
\hline $\begin{array}{l}34 \\
(02)\end{array}$ & $\begin{array}{l}26 \\
(04)\end{array}$ & $\begin{array}{l}21 \\
(05)\end{array}$ & $\begin{array}{l}17 \\
(02)\end{array}$ & $\begin{array}{l}15 \\
(05)\end{array}$ & $\begin{array}{l}13 \\
(04)\end{array}$ & $\begin{array}{l}12 \\
(08)\end{array}$ & $\begin{array}{l}11 \\
(10)\end{array}$ & $\begin{array}{l}10 \\
(10)\end{array}$ & $\begin{array}{l}09 \\
(08)\end{array}$ & $\begin{array}{l}08 \\
(04)\end{array}$ & $\begin{array}{l}08 \\
(12)\end{array}$ & $\begin{array}{l}07 \\
(05)\end{array}$ & $\begin{array}{l}07 \\
(12)\end{array}$ & $\begin{array}{l}06 \\
(02)\end{array}$ & $\begin{array}{l}06 \\
(08)\end{array}$ & $\begin{array}{l}06 \\
(14)\end{array}$ & $\begin{array}{l}06 \\
\text { (20) }\end{array}$ \\
\hline $\begin{array}{l}33 \\
(01)\end{array}$ & $\begin{array}{l}25 \\
(00)\end{array}$ & $\begin{array}{l}20 \\
(00)\end{array}$ & $\begin{array}{l}16 \\
(04)\end{array}$ & $\begin{array}{l}14 \\
(02)\end{array}$ & $\begin{array}{l}12 \\
(04)\end{array}$ & $\begin{array}{l}11 \\
\text { (01) }\end{array}$ & $\begin{array}{l}10 \\
(00)\end{array}$ & $\begin{array}{l}09 \\
\text { (01) }\end{array}$ & $\begin{array}{l}08 \\
(04)\end{array}$ & (09) & $\begin{array}{l}07 \\
(02)\end{array}$ & $\begin{array}{l}06 \\
(10)\end{array}$ & $\begin{array}{l}06 \\
(04)\end{array}$ & $\begin{array}{l}05 \\
(15)\end{array}$ & $\begin{array}{l}05 \\
\text { (10) }\end{array}$ & $\begin{array}{l}05 \\
\text { (05) }\end{array}$ & $\begin{array}{l}05 \\
(00)\end{array}$ \\
\hline $\begin{array}{l}32 \\
(04)\end{array}$ & $\begin{array}{l}24 \\
(04)\end{array}$ & $\begin{array}{l}19 \\
(05)\end{array}$ & $\begin{array}{l}15 \\
(10)\end{array}$ & $\begin{array}{l}13 \\
(09)\end{array}$ & $\begin{array}{l}11 \\
(12)\end{array}$ & $\begin{array}{l}10 \\
(10)\end{array}$ & $\begin{array}{l}09 \\
(10)\end{array}$ & $\begin{array}{l}08 \\
(12)\end{array}$ & $\begin{array}{l}07 \\
(16)\end{array}$ & $\begin{array}{l}06 \\
(22)\end{array}$ & $\begin{array}{l}06 \\
(16)\end{array}$ & $\begin{array}{l}05 \\
(25)\end{array}$ & $\begin{array}{l}05 \\
(20)\end{array}$ & $\begin{array}{l}04 \\
(32)\end{array}$ & $\begin{array}{l}04 \\
(28)\end{array}$ & $\begin{array}{l}04 \\
(24)\end{array}$ & $\begin{array}{l}04 \\
(20)\end{array}$ \\
\hline $\begin{array}{l}31 \\
(07)\end{array}$ & $\begin{array}{l}23 \\
(08)\end{array}$ & $\begin{array}{l}18 \\
(10)\end{array}$ & $\begin{array}{l}14 \\
(16)\end{array}$ & $\begin{array}{l}12 \\
(16)\end{array}$ & $\begin{array}{l}10 \\
(20)\end{array}$ & $\begin{array}{l}09 \\
(19)\end{array}$ & $\begin{array}{l}08 \\
(20)\end{array}$ & $\begin{array}{l}07 \\
(23)\end{array}$ & $\begin{array}{l}06 \\
(28)\end{array}$ & $\begin{array}{l}05 \\
(35)\end{array}$ & $\begin{array}{l}05 \\
(30)\end{array}$ & $\begin{array}{l}04 \\
(40)\end{array}$ & $\begin{array}{l}04 \\
(36)\end{array}$ & $\begin{array}{l}03 \\
(49)\end{array}$ & $\begin{array}{l}03 \\
(46)\end{array}$ & $\begin{array}{l}03 \\
(43)\end{array}$ & $\begin{array}{l}03 \\
(40)\end{array}$ \\
\hline $\begin{array}{l}30 \\
(10)\end{array}$ & $\begin{array}{l}22 \\
(12)\end{array}$ & $\begin{array}{l}17 \\
(15)\end{array}$ & $\begin{array}{l}13 \\
(22)\end{array}$ & $\begin{array}{l}11 \\
(23)\end{array}$ & $\begin{array}{l}09 \\
(28)\end{array}$ & $\begin{array}{l}08 \\
(28)\end{array}$ & $\begin{array}{l}07 \\
(30)\end{array}$ & $\begin{array}{l}06 \\
(34)\end{array}$ & $\begin{array}{l}05 \\
(40)\end{array}$ & $\begin{array}{l}04 \\
(48)\end{array}$ & $\begin{array}{l}04 \\
(44)\end{array}$ & $\begin{array}{l}03 \\
\text { (55) }\end{array}$ & $\begin{array}{l}03 \\
(52)\end{array}$ & $\begin{array}{l}02 \\
(66)\end{array}$ & $\begin{array}{l}02 \\
(64)\end{array}$ & $\begin{array}{l}02 \\
(62)\end{array}$ & $\begin{array}{l}02 \\
(60)\end{array}$ \\
\hline $\begin{array}{l}29 \\
(13)\end{array}$ & $\begin{array}{l}21 \\
(16)\end{array}$ & $\begin{array}{l}16 \\
(20)\end{array}$ & $\begin{array}{l}12 \\
(28)\end{array}$ & $\begin{array}{l}10 \\
(30)\end{array}$ & $\begin{array}{l}08 \\
(36)\end{array}$ & $\begin{array}{l}07 \\
(37)\end{array}$ & $\begin{array}{l}06 \\
(40)\end{array}$ & $\begin{array}{l}05 \\
\text { (45) }\end{array}$ & $\begin{array}{l}04 \\
(52)\end{array}$ & $\begin{array}{l}03 \\
\text { (61) }\end{array}$ & $\begin{array}{l}03 \\
(58)\end{array}$ & $\begin{array}{l}02 \\
(70)\end{array}$ & $\begin{array}{l}02 \\
(68)\end{array}$ & $\begin{array}{l}01 \\
\text { (83) }\end{array}$ & $\begin{array}{l}01 \\
(82)\end{array}$ & $\begin{array}{l}01 \\
(81)\end{array}$ & $\begin{array}{l}01 \\
(80)\end{array}$ \\
\hline
\end{tabular}


The CICT IOU Reference Framework for Stronger AMS System Simulation in Science and Industry

\section{OECS Manifold RTL \& LTR Operators}

$1 /(\mathrm{N}-1)$

RTL Upscale Operator

$$
\sum_{k=1}^{\infty}\left(\frac{1}{N}\right)^{k}=\frac{1}{N-1}, k=1,2,3, \ldots \in N
$$

$1 /(\mathrm{N}+1)$

LTR Downscale Operator

$$
\sum_{k=0}^{\infty}(-1)^{k}\left(\frac{1}{N}\right)^{(k+1)}=\frac{1}{N+1}, k=0,1,2,3, \ldots \in N
$$

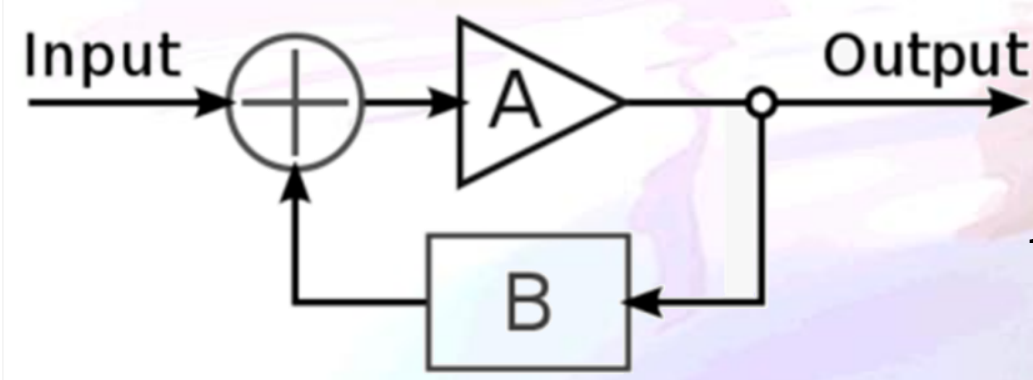

$A=1 / N$
$B=1$

42 Rodolfo A. Fiorini

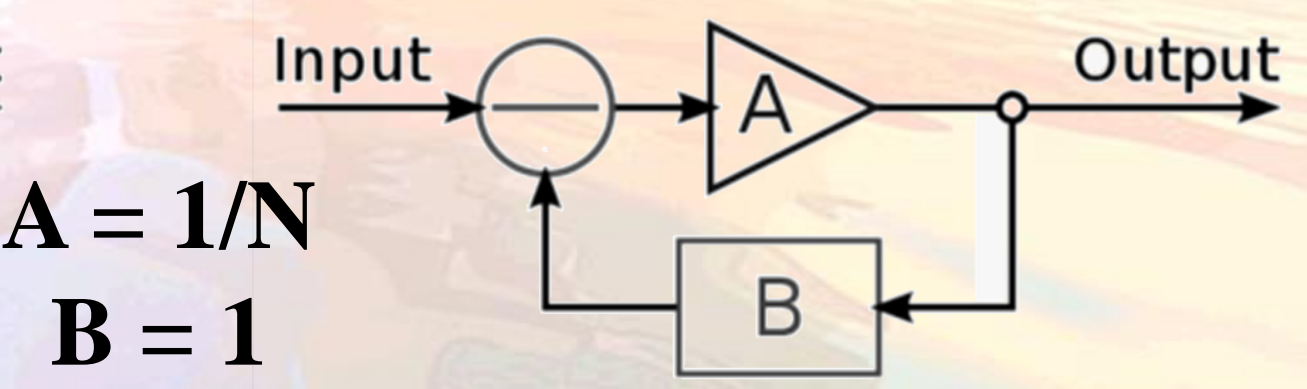

POLITECNICO MILANO 1863 
The CICT IOU Reference Framework for Stronger AMS System Simulation in Science and Industry Hunum

\section{OECS Manifold Two Basic Reflexion Types}

\section{Incidence $(\Omega)$}

vs.

Correspondence $(U)$ 


\section{The CICT IOU Reference Framework for Stronger AMS System Simulation in Science and Industry}

\section{Incidence $(\cap)$}

\begin{tabular}{|c|c|c|c|c|c|c|c|c|c|c|c|c|c|c|c|c|c|}
\hline $\begin{array}{l}1 / \\
03\end{array}$ & $\begin{array}{l}1 / \\
04\end{array}$ & $\begin{array}{l}1 / \\
05\end{array}$ & (06) & $\begin{array}{l}1 / \\
07\end{array}$ & $\begin{array}{l}1 / \\
08\end{array}$ & $\begin{array}{l}1 / \\
09\end{array}$ & $\begin{array}{l}1 / \\
10\end{array}$ & $\begin{array}{l}1 / \\
11\end{array}$ & $\begin{array}{l}1 / \\
12\end{array}$ & $\begin{array}{l}1 / \\
13\end{array}$ & $\frac{11}{14}$ & $\begin{array}{l}1 / \\
15\end{array}$ & $\begin{array}{l}1 / \\
16\end{array}$ & $\begin{array}{l}1 / \\
17\end{array}$ & $\begin{array}{l}1 / \\
18\end{array}$ & $\begin{array}{l}1 / \\
19\end{array}$ & $\begin{array}{l}1 / \\
20\end{array}$ \\
\hline $\begin{array}{l}37 \\
(11)\end{array}$ & $\begin{array}{l}29 \\
(16)\end{array}$ & $\begin{array}{l}24 \\
(20)\end{array}$ & $\begin{array}{l}20 \\
(20)\end{array}$ & $\begin{array}{l}18 \\
(26)\end{array}$ & $\begin{array}{l}16 \\
(28)\end{array}$ & $\begin{array}{l}15 \\
\text { (35) }\end{array}$ & $\begin{array}{l}14 \\
(40)\end{array}$ & $\begin{array}{l}13 \\
(43)\end{array}$ & $\begin{array}{l}12 \\
(44)\end{array}$ & $\begin{array}{l}11 \\
(43)\end{array}$ & $\begin{array}{l}11 \\
(54)\end{array}$ & $\begin{array}{l}10 \\
(50)\end{array}$ & $\begin{array}{l}10 \\
(60)\end{array}$ & $\begin{array}{l}09 \\
(53)\end{array}$ & $\begin{array}{l}09 \\
(62)\end{array}$ & $\begin{array}{l}09 \\
(71)\end{array}$ & $\begin{array}{l}09 \\
(80)\end{array}$ \\
\hline $\begin{array}{l}36 \\
(08)\end{array}$ & $\begin{array}{l}28 \\
(12)\end{array}$ & $\begin{array}{l}23 \\
(15)\end{array}$ & $\begin{array}{l}19 \\
(14)\end{array}$ & $\begin{array}{l}17 \\
\text { (19) }\end{array}$ & $\begin{array}{l}15 \\
(20)\end{array}$ & $\begin{array}{l}14 \\
(26)\end{array}$ & $\begin{array}{l}13 \\
(30)\end{array}$ & $\begin{array}{l}12 \\
(32)\end{array}$ & $\begin{array}{l}11 \\
(32)\end{array}$ & $\begin{array}{l}10 \\
(30)\end{array}$ & $\begin{array}{l}10 \\
(40)\end{array}$ & $\begin{array}{l}09 \\
\text { (35) }\end{array}$ & $\begin{array}{l}09 \\
(44)\end{array}$ & $\begin{array}{l}08 \\
(36)\end{array}$ & $\begin{array}{l}08 \\
(44)\end{array}$ & $\begin{array}{l}08 \\
(52)\end{array}$ & $\begin{array}{l}08 \\
(60)\end{array}$ \\
\hline $\begin{array}{l}35 \\
(05)\end{array}$ & $\begin{array}{l}27 \\
(08)\end{array}$ & $\begin{array}{l}22 \\
(10)\end{array}$ & $\begin{array}{l}18 \\
(08)\end{array}$ & $\begin{array}{l}16 \\
\text { (12 }\end{array}$ & $\int_{(12)}^{14-}$ & $\begin{array}{l}-13- \\
\text { (17) }\end{array}$ & $\begin{array}{l}12- \\
(20)\end{array}$ & $\begin{array}{l}-11- \\
(21)\end{array}$ & $\begin{array}{l}10- \\
(20)\end{array}$ & $\begin{array}{l}09 \\
(17)\end{array}$ & $\begin{array}{l}09 \\
(26)\end{array}$ & $\begin{array}{l}08 \\
(20)\end{array}$ & $\begin{array}{l}08 \\
(28)\end{array}$ & $\begin{array}{l}07 \\
(19)\end{array}$ & $\begin{array}{l}07 \\
(26)\end{array}$ & $\begin{array}{l}07 \\
\text { (33) }\end{array}$ & $\begin{array}{l}07 \\
(40)\end{array}$ \\
\hline $\begin{array}{l}34 \\
\text { (02) }\end{array}$ & $\begin{array}{l}26 \\
(04)\end{array}$ & $\begin{array}{l}21 \\
(05)\end{array}$ & $\begin{array}{l}17 \\
(02)\end{array}$ & $\begin{array}{l}15 / \\
(05)\end{array}$ & $\begin{array}{l}13 \\
(04)\end{array}$ & $\begin{array}{l}12 \\
(08)\end{array}$ & $\begin{array}{l}11 \\
(10)\end{array}$ & $\begin{array}{l}10 \\
(10)\end{array}$ & $\begin{array}{l}09 \\
(08)\end{array}$ & $\begin{array}{l}08 \\
(04)\end{array}$ & $\begin{array}{l}08- \\
\text { (12) }\end{array}$ & $\begin{array}{l}-07 \\
(05)\end{array}$ & $\begin{array}{l}07 \\
(12)\end{array}$ & $\begin{array}{l}06 \\
(02)\end{array}$ & $\begin{array}{l}06 \\
(08)\end{array}$ & $\begin{array}{l}06 \\
(14)\end{array}$ & $\begin{array}{l}06 \\
(20)\end{array}$ \\
\hline $\begin{array}{l}33 \\
\text { (01) }\end{array}$ & $\begin{array}{l}25 \\
(00)\end{array}$ & $\begin{array}{l}20 \\
(00)\end{array}$ & $\begin{array}{l}16 \\
(04)\end{array}$ & 1 & $/_{(04)}^{12-}$ & $\begin{array}{l}-11- \\
\text { (01) }\end{array}$ & $\begin{array}{l}10- \\
(00)\end{array}$ & $\begin{array}{l}-09- \\
(01)\end{array}$ & $\begin{array}{l}08- \\
(04)\end{array}$ & $\begin{array}{l}07 \\
(09)\end{array}$ & $\begin{array}{l}07 \\
(02)\end{array}$ & $\begin{array}{l}06 \\
(10)\end{array}$ & $\begin{array}{l}06 \\
(04)\end{array}$ & $\begin{array}{l}05 \\
(15)\end{array}$ & $\begin{array}{l}05 \\
(10)\end{array}$ & $\begin{array}{l}05 \\
(05)\end{array}$ & $\begin{array}{l}05 \\
(00)\end{array}$ \\
\hline $\begin{array}{l}32 \\
(04)\end{array}$ & $\begin{array}{l}24 \\
(04)\end{array}$ & $\begin{array}{l}19 \\
(05)\end{array}$ & 15 & b) & $\begin{array}{l}11 \\
(12)\end{array}$ & $\begin{array}{l}10 \\
(10)\end{array}$ & $\begin{array}{l}09 \\
(10)\end{array}$ & $\begin{array}{l}08 \\
(12)\end{array}$ & $\begin{array}{l}07 \\
(16)\end{array}$ & $\begin{array}{l}06 \\
(22)\end{array}$ & $\begin{array}{l}d 6 \\
\text { (16) }\end{array}$ & $\begin{array}{l}05 \\
(25)\end{array}$ & $\begin{array}{l}05 \\
(20)\end{array}$ & $\begin{array}{l}04 \\
\text { (32) }\end{array}$ & $\begin{array}{l}04 \\
(28)\end{array}$ & $\begin{array}{l}04 \\
(24)\end{array}$ & $\begin{array}{l}04 \\
(20)\end{array}$ \\
\hline $\begin{array}{l}31 \\
\text { (07) }\end{array}$ & $\begin{array}{l}23 \\
(08)\end{array}$ & $\begin{array}{l}18 \\
(10)\end{array}$ & $\begin{array}{l}14 \\
(16)\end{array}$ & 12 & $\begin{array}{l}10- \\
(20)\end{array}$ & $\begin{array}{l}09-1 \\
\text { (19) }\end{array}$ & $\begin{array}{l}08 \\
(20)\end{array}$ & $\begin{array}{l}-07- \\
(23)\end{array}$ & $\begin{array}{l}-06 \\
(28)\end{array}$ & $\begin{array}{l}05 \\
\text { (35) }\end{array}$ & $\begin{array}{l}05 \\
(30)\end{array}$ & $\begin{array}{l}04 \\
(40)\end{array}$ & $\begin{array}{l}04 \\
(36)\end{array}$ & $\begin{array}{l}03 \\
(49)\end{array}$ & $\begin{array}{l}03 \\
(46)\end{array}$ & $\begin{array}{l}03 \\
(43)\end{array}$ & $\begin{array}{l}03 \\
(40)\end{array}$ \\
\hline $\begin{array}{l}30 \\
(10)\end{array}$ & $\begin{array}{l}22 \\
(12)\end{array}$ & $\begin{array}{l}17 \\
(15)\end{array}$ & $\begin{array}{l}13 \\
(22)\end{array}$ & $/(23)$ & $\begin{array}{l}09 \\
(28)\end{array}$ & $\begin{array}{l}08 \\
(28)\end{array}$ & $\begin{array}{l}07 \\
(30)\end{array}$ & $\begin{array}{l}06 \\
(34)\end{array}$ & $\begin{array}{l}05 \\
(40)\end{array}$ & $\begin{array}{l}04 \\
(48)\end{array}$ & $\begin{array}{l}04 \\
(44)\end{array}$ & $\begin{array}{l}03 \\
(55)\end{array}$ & $\begin{array}{l}03 \\
(52)\end{array}$ & $\begin{array}{l}02 \\
(66)\end{array}$ & $\begin{array}{l}02 \\
(64)\end{array}$ & $\begin{array}{l}02 \\
(62)\end{array}$ & $\begin{array}{l}02 \\
(60)\end{array}$ \\
\hline $\begin{array}{l}29 \\
\text { (13) }\end{array}$ & $\begin{array}{l}21 \\
(16)\end{array}$ & $\begin{array}{l}16 \\
(20)\end{array}$ & $\begin{array}{l}12 \\
(28)\end{array}$ & $\begin{array}{l}10 \\
(30)\end{array}$ & $\begin{array}{l}08 \\
(36)\end{array}$ & $\begin{array}{l}07 \\
\text { (37) }\end{array}$ & $\begin{array}{l}06 \\
(40)\end{array}$ & $\begin{array}{l}05 \\
(45)\end{array}$ & $\begin{array}{l}04 \\
(52)\end{array}$ & $\begin{array}{l}03 \\
(61)\end{array}$ & $\begin{array}{l}03 \\
(58)\end{array}$ & $\begin{array}{l}02 \\
(70)\end{array}$ & $\begin{array}{l}02 \\
(68)\end{array}$ & $\begin{array}{l}01 \\
(83)\end{array}$ & $\begin{array}{l}01 \\
(82)\end{array}$ & $\begin{array}{l}01 \\
(81)\end{array}$ & $\begin{array}{l}01 \\
(80)\end{array}$ \\
\hline
\end{tabular}




\section{The CICT IOU Reference Framework for Stronger AMS System Simulation in Science and Industry}

\section{Correspondence $(U)$}

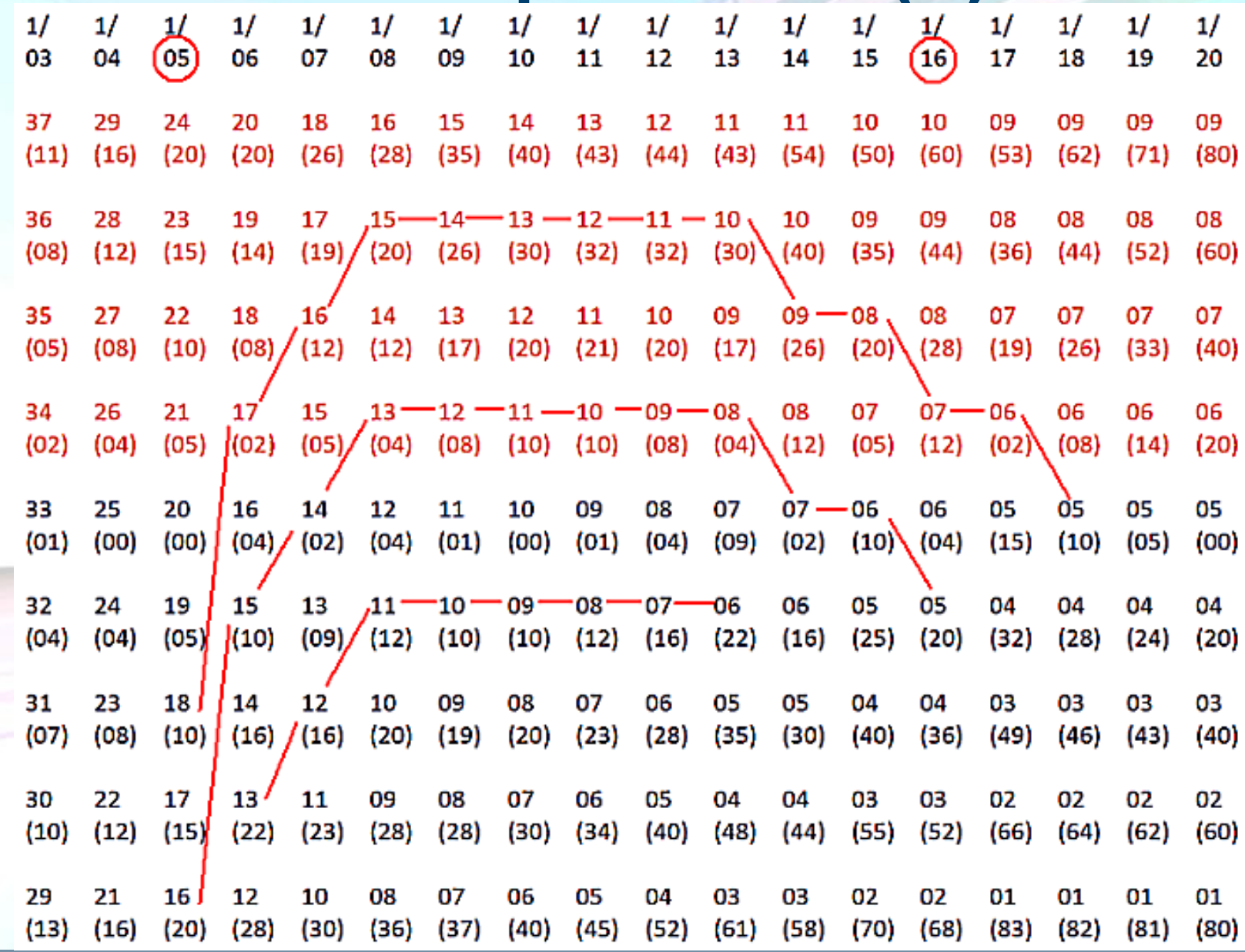




\section{The CICT IOU Reference Framework for Stronger AMS System Simulation in Science and Industry}

Ans

\section{Incidence-Correspondence Alternation}

\begin{tabular}{|c|c|c|c|c|c|c|c|c|c|c|c|c|c|c|c|c|c|}
\hline 1/ & $1 /$ & $1 /$ & $1 /$ & $1 /$ & $1 /$ & $1 /$ & $1 /$ & $1 /$ & $1 /$ & $1 /$ & $1 /$ & $1 /$ & $1 /$ & $1 /$ & $1 /$ & $1 /$ & $1 /$ \\
\hline 03 & 04 & 05 & 06 & 07 & 08 & 09 & 10 & 11 & 12 & 13 & 14 & 15 & 16 & 17 & 18 & 19 & 20 \\
\hline (11) & $\begin{array}{l}29 \\
(16)\end{array}$ & $\begin{array}{l}24 \\
(20)\end{array}$ & $\begin{array}{l}20 \\
(20)\end{array}$ & $\begin{array}{l}18 \\
(26)\end{array}$ & $\begin{array}{l}16 \\
(28)\end{array}$ & $\begin{array}{l}15 \\
(35)\end{array}$ & $\begin{array}{l}14 \\
(40)\end{array}$ & $\begin{array}{l}13 \\
(43)\end{array}$ & $\begin{array}{l}12 \\
(44)\end{array}$ & $\begin{array}{l}11 \\
(43)\end{array}$ & $\begin{array}{l}11 \\
(54)\end{array}$ & $\begin{array}{l}10 \\
(50)\end{array}$ & $\begin{array}{l}10 \\
(60)\end{array}$ & $\begin{array}{l}09 \\
(53)\end{array}$ & $\begin{array}{l}09 \\
(62)\end{array}$ & $\begin{array}{l}09 \\
(71)\end{array}$ & $\begin{array}{l}09 \\
(80)\end{array}$ \\
\hline 8) & $\begin{array}{l}28 \\
(12)\end{array}$ & $\begin{array}{l}23 \\
(15)\end{array}$ & $\begin{array}{l}19 \\
(14)\end{array}$ & $\begin{array}{l}17 \\
(19)\end{array}$ & $\begin{array}{l}15 \\
(20)\end{array}$ & $\begin{array}{l}14 \\
(26)\end{array}$ & $\begin{array}{l}13 \\
(30)\end{array}$ & $\begin{array}{l}12 \\
(32)\end{array}$ & $\begin{array}{l}11 \\
(32)\end{array}$ & $\begin{array}{l}10 \\
(30)\end{array}$ & $\begin{array}{l}10 \\
(40)\end{array}$ & $\begin{array}{l}09 \\
\text { (35) }\end{array}$ & $\begin{array}{l}09 \\
(44)\end{array}$ & $\begin{array}{l}08 \\
(36)\end{array}$ & $\begin{array}{l}08 \\
(44)\end{array}$ & $\begin{array}{l}08 \\
(52)\end{array}$ & $\begin{array}{l}08 \\
(60)\end{array}$ \\
\hline 1 & $\begin{array}{l}27 \\
(08)\end{array}$ & $\begin{array}{l}22 \\
(10)\end{array}$ & $\begin{array}{l}18 \\
(08)\end{array}$ & $\begin{array}{l}16 \\
(12)\end{array}$ & $\begin{array}{l}14 \\
(12)\end{array}$ & $\begin{array}{l}13 \\
(17)\end{array}$ & $\begin{array}{l}12 \\
(20)\end{array}$ & $\begin{array}{l}11 \\
(21)\end{array}$ & $\begin{array}{l}10 \\
(20)\end{array}$ & $\begin{array}{l}09 \\
(17)\end{array}$ & $\begin{array}{l}09 \\
(26)\end{array}$ & $\begin{array}{l}08 \\
(20)\end{array}$ & $\begin{array}{l}08 \\
(28)\end{array}$ & $\begin{array}{l}07 \\
(19)\end{array}$ & $\begin{array}{l}07 \\
(26)\end{array}$ & $\begin{array}{l}07 \\
(33)\end{array}$ & $\begin{array}{l}07 \\
(40)\end{array}$ \\
\hline ) & $\begin{array}{l}26 \\
(04)\end{array}$ & $\begin{array}{l}21 \\
(05)\end{array}$ & $\begin{array}{l}17 \\
(02)\end{array}$ & $\begin{array}{l}15 \\
(05)\end{array}$ & $\begin{array}{l}13 \\
(04)\end{array}$ & $\begin{array}{l}12 \\
(08)\end{array}$ & $\begin{array}{l}11 \\
(10)\end{array}$ & $\begin{array}{l}10 \\
(10)\end{array}$ & $\begin{array}{l}09 \\
(08)\end{array}$ & $\begin{array}{l}08 \\
(04)\end{array}$ & $\begin{array}{l}08 \\
(12)\end{array}$ & $\begin{array}{l}07 \\
(05)\end{array}$ & $\begin{array}{l}07 \\
(12)\end{array}$ & $\begin{array}{l}06 \\
(02)\end{array}$ & $\begin{array}{l}06 \\
(08)\end{array}$ & $\begin{array}{l}06 \\
(14)\end{array}$ & $\begin{array}{l}06 \\
(20)\end{array}$ \\
\hline 1) & $\begin{array}{l}25 \\
(00)\end{array}$ & $\begin{array}{l}20 \\
(00)\end{array}$ & $\begin{array}{l}16 \\
(04)\end{array}$ & $\begin{array}{l}14 \\
(02)\end{array}$ & $\begin{array}{l}12 \\
(04)\end{array}$ & $\begin{array}{l}11 \\
(01)\end{array}$ & $\begin{array}{l}10 \\
(00)\end{array}$ & $\begin{array}{l}09 \\
(01)\end{array}$ & $\begin{array}{l}08 \\
(04)\end{array}$ & $\begin{array}{l}07 \\
(09)\end{array}$ & $\begin{array}{l}07 \\
(02)\end{array}$ & $\begin{array}{l}06 \\
(10)\end{array}$ & $\begin{array}{l}06 \\
(04)\end{array}$ & $\begin{array}{l}05 \\
(15)\end{array}$ & $\begin{array}{l}05 \\
(10)\end{array}$ & $\begin{array}{l}05 \\
(05)\end{array}$ & $\begin{array}{l}05 \\
(00)\end{array}$ \\
\hline 4) & $\begin{array}{l}24 \\
(04)\end{array}$ & $\begin{array}{l}19 \\
(05)\end{array}$ & $\begin{array}{l}15 \\
(10)\end{array}$ & $\begin{array}{l}13 \\
(09)\end{array}$ & $\begin{array}{l}11 \\
(12)\end{array}$ & $\begin{array}{l}10 \\
(10)\end{array}$ & $\begin{array}{l}09 \\
(10)\end{array}$ & $\begin{array}{l}08 \\
(12)\end{array}$ & $\begin{array}{l}07 \\
(16)\end{array}$ & $\begin{array}{l}06 \\
(22)\end{array}$ & $\begin{array}{l}06 \\
(16)\end{array}$ & $\begin{array}{l}05 \\
(25)\end{array}$ & $\begin{array}{l}05 \\
(20)\end{array}$ & $\begin{array}{l}04 \\
(32)\end{array}$ & $\begin{array}{l}04 \\
(28)\end{array}$ & $\begin{array}{l}04 \\
(24)\end{array}$ & $\begin{array}{l}04 \\
(20)\end{array}$ \\
\hline 7) & $\begin{array}{l}23 \\
(08)\end{array}$ & $\begin{array}{l}18 \\
(10)\end{array}$ & $\begin{array}{l}14 \\
(16)\end{array}$ & $\begin{array}{l}12 \\
(16)\end{array}$ & $\begin{array}{l}10 \\
(20)\end{array}$ & $\begin{array}{l}09 \\
(19)\end{array}$ & $\begin{array}{l}08 \\
(20)\end{array}$ & $\begin{array}{l}07 \\
(23)\end{array}$ & $\begin{array}{l}06 \\
(28)\end{array}$ & $\begin{array}{l}05 \\
(35)\end{array}$ & $\begin{array}{l}05 \\
(30)\end{array}$ & $\begin{array}{l}04 \\
(40)\end{array}$ & $\begin{array}{l}04 \\
(36)\end{array}$ & $\begin{array}{l}03 \\
(49)\end{array}$ & $\begin{array}{l}03 \\
(46)\end{array}$ & $\begin{array}{l}03 \\
(43)\end{array}$ & $\begin{array}{l}03 \\
(40)\end{array}$ \\
\hline (10) & $\begin{array}{l}22 \\
(12)\end{array}$ & $\begin{array}{l}17 \\
(15)\end{array}$ & $\begin{array}{l}13 \\
(22)\end{array}$ & $\begin{array}{l}11 \\
(23)\end{array}$ & $\begin{array}{l}09 \\
(28)\end{array}$ & $\begin{array}{l}08 \\
(28)\end{array}$ & $\begin{array}{l}07 \\
(30)\end{array}$ & $\begin{array}{l}06 \\
(34)\end{array}$ & $\begin{array}{l}05 \\
(40)\end{array}$ & $\begin{array}{l}04 \\
(48)\end{array}$ & $\begin{array}{l}04 \\
(44)\end{array}$ & $\begin{array}{l}03 \\
(55)\end{array}$ & $\begin{array}{l}03 \\
(52)\end{array}$ & $\begin{array}{l}02 \\
(66)\end{array}$ & $\begin{array}{l}02 \\
(64)\end{array}$ & $\begin{array}{l}02 \\
(62)\end{array}$ & $\begin{array}{l}02 \\
(60)\end{array}$ \\
\hline 3) & $\begin{array}{l}21 \\
(16)\end{array}$ & $\begin{array}{l}16 \\
(20)\end{array}$ & $\begin{array}{l}12 \\
(28)\end{array}$ & $\begin{array}{l}10 \\
(30)\end{array}$ & $\begin{array}{l}08 \\
(36)\end{array}$ & $\begin{array}{l}07 \\
(37)\end{array}$ & $\begin{array}{l}06 \\
(40)\end{array}$ & $\begin{array}{l}05 \\
(45)\end{array}$ & $\begin{array}{l}04 \\
(52)\end{array}$ & $\begin{array}{l}03 \\
(61)\end{array}$ & $\begin{array}{l}03 \\
(58)\end{array}$ & $\begin{array}{l}02 \\
(70)\end{array}$ & $\begin{array}{l}02 \\
(68)\end{array}$ & $\begin{array}{l}01 \\
(83)\end{array}$ & $\begin{array}{l}01 \\
(82)\end{array}$ & $\begin{array}{l}01 \\
(81)\end{array}$ & $\begin{array}{l}01 \\
(80)\end{array}$ \\
\hline
\end{tabular}




\section{The CICT IOU Reference Framework for Stronger AMS System Simulation in Science and Industry}

\section{Solid Number $\left(\mathrm{SN}_{\mathrm{x}}\right)$}

Quite recently, the scientific community has acquired new awareness about traditional rational number system $\boldsymbol{Q}$ properties. It can be regarded as a highly sophisticated open logic, powerful and flexible LTR and RTL formal language, with self-defining consistent words and rules, starting from elementary generators and relations.

To face the challenge of complex system modeling (AMS system modeling), we need to be able to control system uncertainty quantification from macroscale, through mesoscale, till nanoscale and beyond.

This new awareness can guide the development of new competitive algorithm and application. Specifically, knowledge about the fundamental operative difference of :

a) "Symbolic vs. OpeRational Number Representation" and

b) "Prime vs. Primitive Number" or "Solid Number" $\left(\mathrm{SN}_{\mathrm{x}}\right)$.

$\mathrm{SN}_{\mathrm{x}}$ can play a fundamental role by capturing and optimally encoding deterministic information by RFD (Representation Fundamental Domain). 
The CICT IOU Reference Framework for Stronger AMS System Simulation in Science and Industry

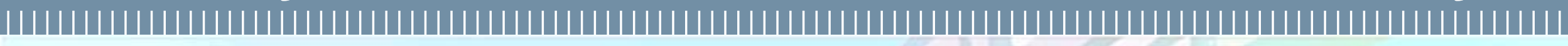

\section{Solid Number $\left(\mathrm{SN}_{\mathbf{x}}\right)$}

\section{Prime}

VS.

Primitive Number

Solid Number $\left(\mathbf{S N}_{\mathrm{x}}\right)$ 
The CICT IOU Reference Framework for Stronger AMS System Simulation in Science and Industry

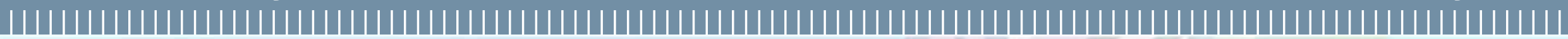

\section{RFD R $\mathrm{L}_{\mathrm{L}}$ LTR-RTL}

\section{Inner Linear Coordinate Reference (ILCR) for SN 7}

\begin{tabular}{|l|l|l|l|l|l|l|l|l|l|l|l|l|l|}
\hline$\Longleftrightarrow$ & 5 & 4 & 3 & 2 & 1 & 0 & 5 & 4 & 3 & 2 & 1 & 0 & RTL \\
\hline LTR & 1 & 2 & 3 & 4 & 5 & 6 & 1 & 2 & 3 & 4 & 5 & 6 & $\Longrightarrow$ \\
\hline & & & & & & & & & & & & & \\
\hline $\mathbf{Q}_{\mathbf{L}}$ & 1 & 4 & 2 & 8 & 5 & 7 & 1 & 4 & 2 & 8 & 5 & 7 & \\
\hline $\mathbf{R}_{\mathbf{L}}$ & $\mathbf{3}$ & $\mathbf{2}$ & $\mathbf{6}$ & $\mathbf{4}$ & $\mathbf{5}$ & $\mathbf{1}$ & $\mathbf{3}$ & $\mathbf{2}$ & $\mathbf{6}$ & $\mathbf{4}$ & $\mathbf{5}$ & $\mathbf{1}$ & \\
\hline
\end{tabular}

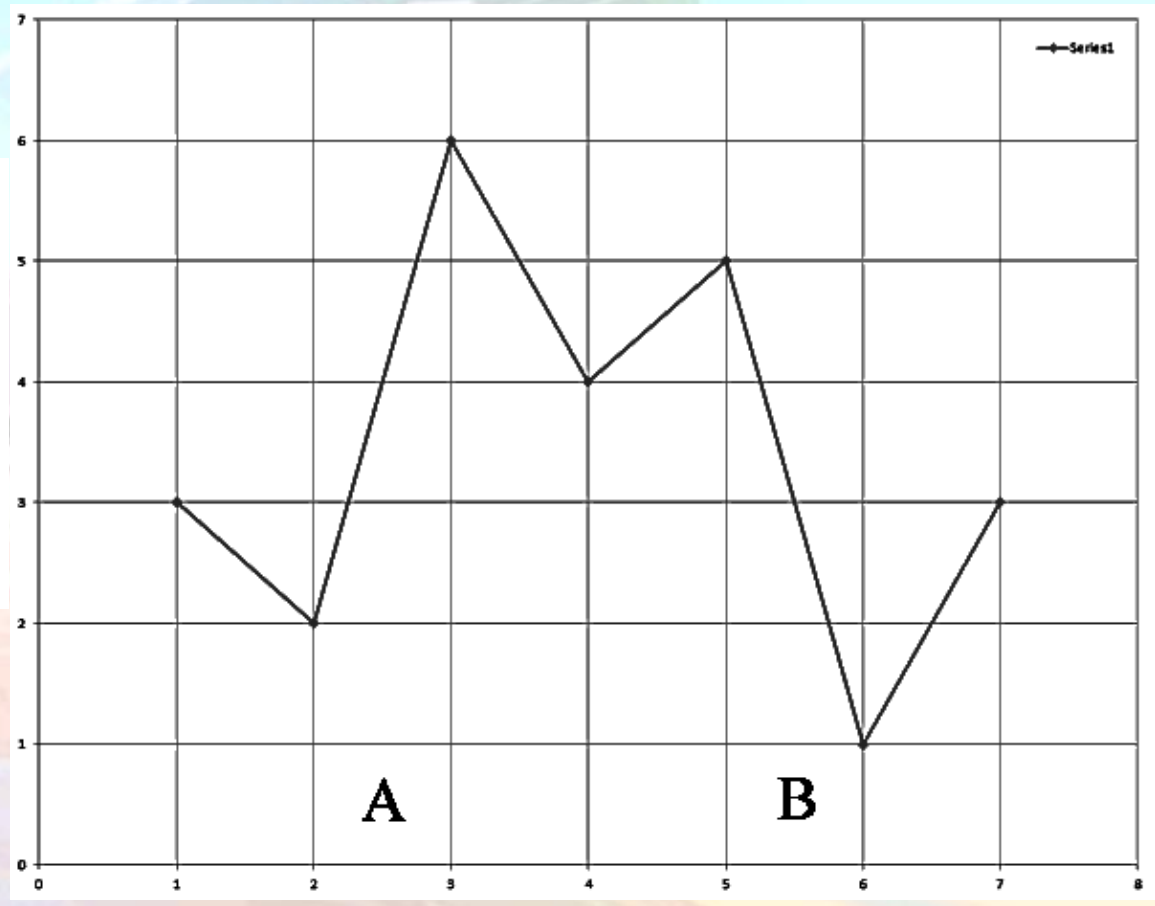

(R.A. Fiorini, 2013) 
The CICT IOU Reference Framework for Stronger AMS System Simulation in Science and Industry

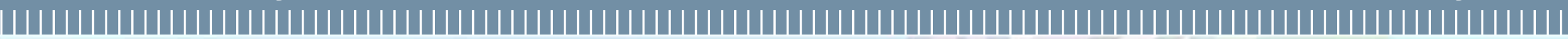

\section{RFD R $\mathbf{L}_{\text {LTR-RTL }}$}

\section{Inner Linear Coordinate Reference (ILCR) for SN 7}
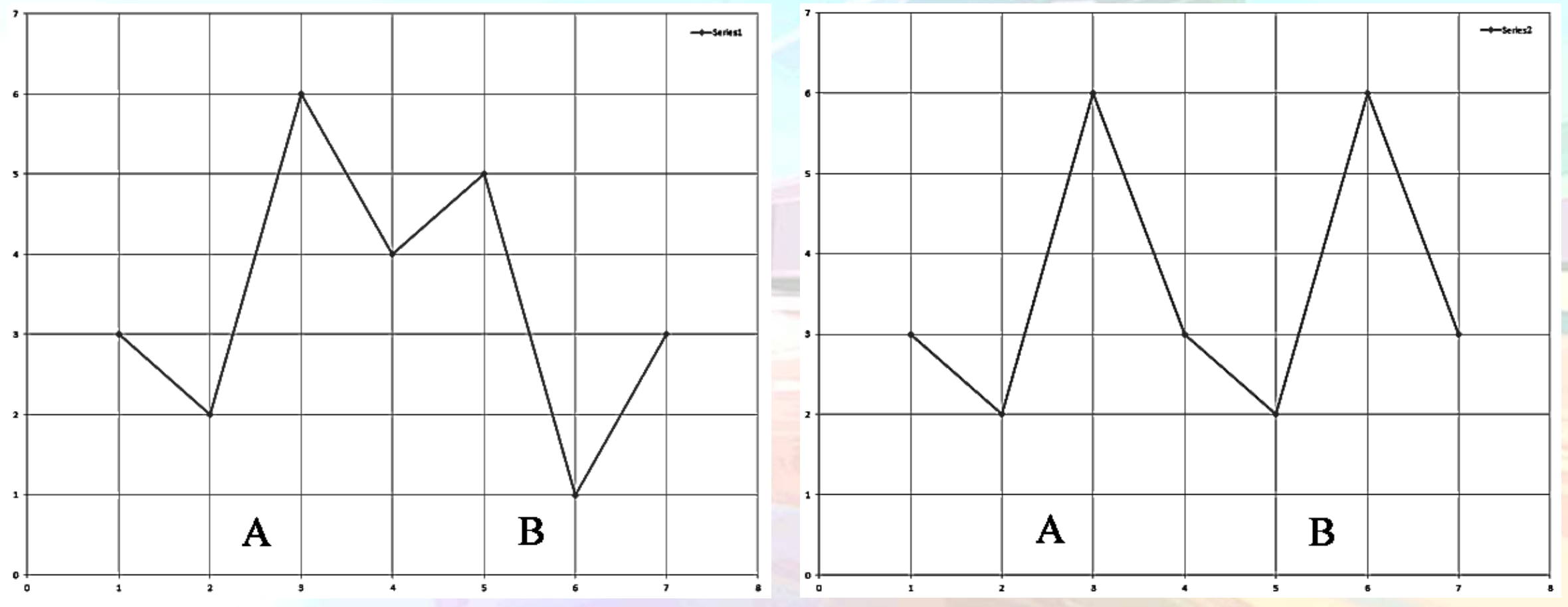

(R.A. Fiorini, 2013)

50 Rodolfo A. Fiorini 


\section{The CICT IOU Reference Framework for Stronger AMS System Simulation in Science and Industry}

\section{Solid Number $\left(\mathrm{SN}_{\mathrm{x}}\right)$}

Let us introduce a convenient LTR symbolic compression operator $(\mathrm{SCO})$ as:

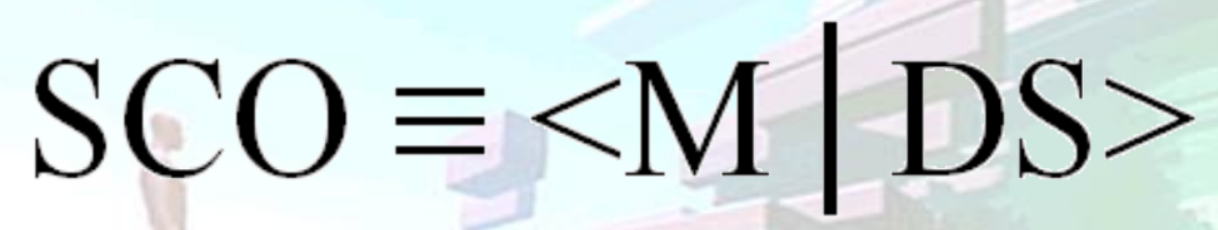

where DS is a finite digit string of length $L$ and $\mathbf{M}$ is the number of times DS is repeated to get our unfolded digit string in full, e.g.:

$(4 \mid 1) \equiv 1111$ or $(2 \mid 123) \equiv 123123)$

Usual symbolic string operations can be applied to SCO. 


\section{The CICT IOU Reference Framework for Stronger AMS System Simulation in Science and Industry}

\section{Solid Number $\left(\mathrm{SN}_{\mathrm{x}}\right)$}

According to our SCO approach, the correct coherent relation representation of traditional scalar modulus $D=7$, as denominator of Egyptian fraction, is given by:

$\left|C Q_{1}=\frac{1}{C D_{1}} \equiv \frac{1}{\langle\infty \mid(<\infty|0\rangle\langle 1| 7>)\rangle} \equiv 0.\right|\langle\infty|R F D(7)>\equiv 0 .<\infty| 142857\rangle$

To conserve the full information content of rational correspondence at higher level, we realize that we have to take into account not only the usual modulus information, but even the related periodic precision length information $\boldsymbol{W}=\mathbf{6}$ (numeric period or external phase representation) in this case (i.e. $C D_{l}=000007$ as base $R F D$ ). 


\section{The CICT IOU Reference Framework for Stronger AMS System Simulation in Science and Industry}

\section{Solid Number $\left(\mathrm{SN}_{\mathrm{x}}\right)$}

We can use Euler's identity to establish the usual fundamental relationship between trigonometric functions and the subalgebra of complex numbers of the geometric algebra $\mathrm{Cl}_{2}$ in the following way:

$$
\exp \left(\alpha e_{12}\right)=\cos \alpha+e_{12} \sin \alpha
$$

where $e_{12}$ is the imaginary unit, usually noted as $i=\sqrt{ }-1$.

The final result is:

$$
C Q Q_{1}=\frac{1}{C D D_{1}}=\frac{1}{7} \exp \left(-\frac{\pi(1+2 n)}{3} e_{12}\right)
$$

and

$$
C D D_{1}=\frac{1}{C Q Q_{1}}=7 \exp \left(\frac{\pi(1+2 n)}{3} e_{12}\right)=7\left(\frac{1}{2}+\frac{\sqrt{3}}{2} e_{12}\right)_{(p . v)}
$$

for $n=1,2,3, \ldots$ in $N$, where (p.v.) means "principal value". 


\section{The CICT IOU Reference Framework for Stronger AMS System Simulation in Science and Industry}

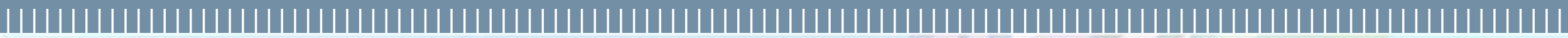

Discrete and Continuous Roots of Unity

Inner - Outer Phased Generators and Relations for SN 7

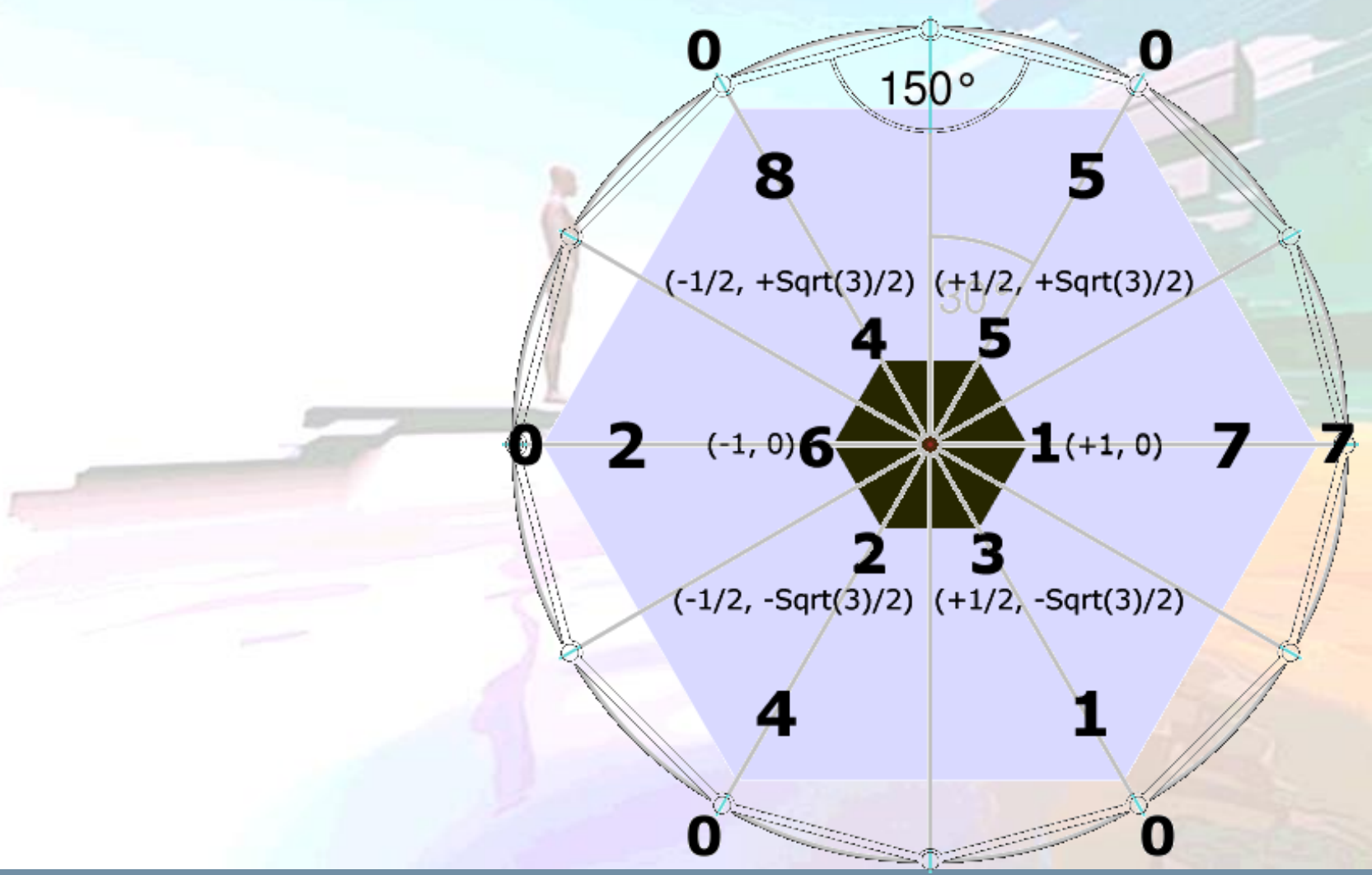

(R.A. Fiorini, 2013)

54 Rodolfo A. Fiorini 


\section{The CICT IOU Reference Framework for Stronger AMS System Simulation in Science and Industry}

\section{Solid Number $\left(\mathrm{SN}_{\mathrm{x}}\right)$}

CICT shows that any natural number $D$ in $N$ has associated a specific, non-arbitrary exterior phase relationship (OECS, Optimized Exponential Cyclic Sequence) coherence information, that we must take into account to full conserve its information content by computation in Euclidean space.

The interested reader will have already guessed the relationship of our result to de Moivre number or primitive root of unity (i.e. any complex number that gives +1.0 when raised to some integer power of $n$ ). In this way, we can exploit Rational numbers $\boldsymbol{Q}$ full information content to get stronger solutions to current AMS system modeling problems. 


\section{The CICT IOU Reference Framework for Stronger AMS System Simulation in Science and Industry}

\section{CICT Computational Processing}

Our knowledge of RFD (Representation Fundamental Domain) $\mathrm{Q}_{\mathrm{L}}$ and corresponding RFD $R_{L}$ can allow reversing LTR numeric power convergent sequence to its corresponding RTL numeric power divergent sequence uniquely.

Reversing a convergent sequence into a divergent one and vice-versa is the fundamental property to reach information conservation, i.e. information reversibility.

Eventually, OECS (Optimized Exponential Cyclic Sequences) have strong connection even to classic DFT algorithmic structure for discrete data, Number-Theoretic Transform (NTT), Laplace and Mellin Transforms.

Coherent precision correspondence leads to transparency, ordering, reversibility, kosmos, simplicity, clarity, and to algorithmic quantum incomputability on real macroscopic machines. 


\section{The CICT IOU Reference Framework for Stronger AMS System Simulation in Science and Industry}

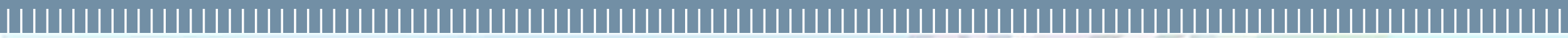

\section{SN 7 Family Group (First Order)}

\section{Remainder-Quotient OECS Recurrence Relation}

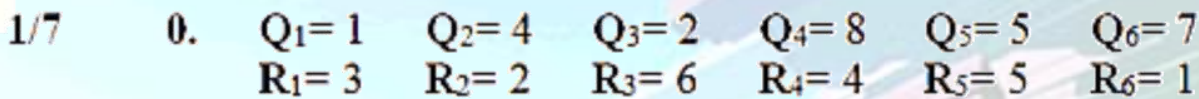

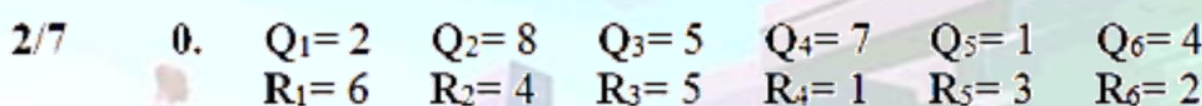

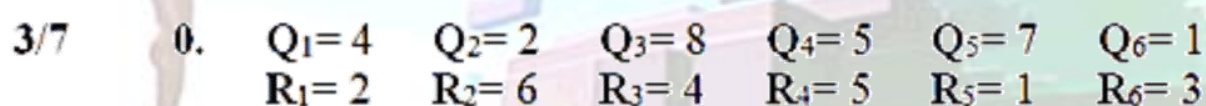

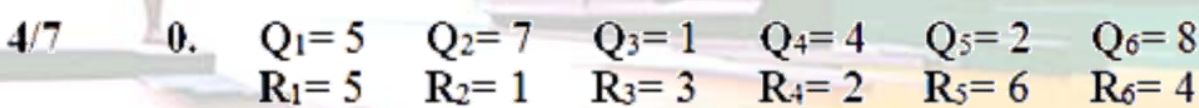

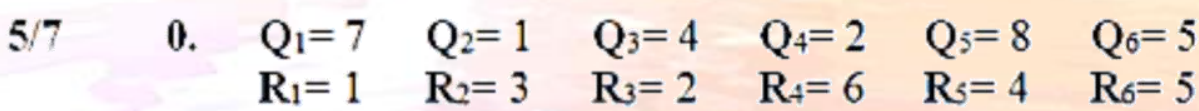

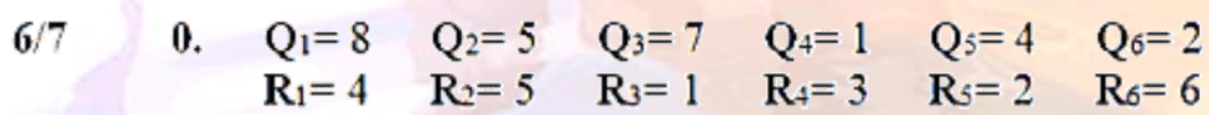

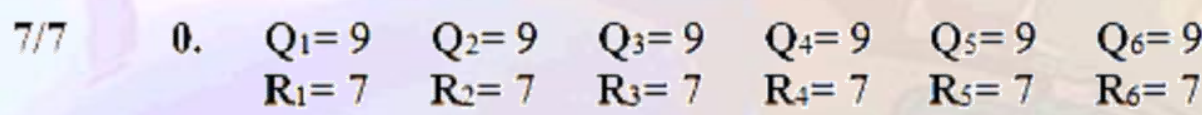

(R.A. Fiorini, 2013) 


\section{The CICT IOU Reference Framework for Stronger AMS System Simulation in Science and Industry}

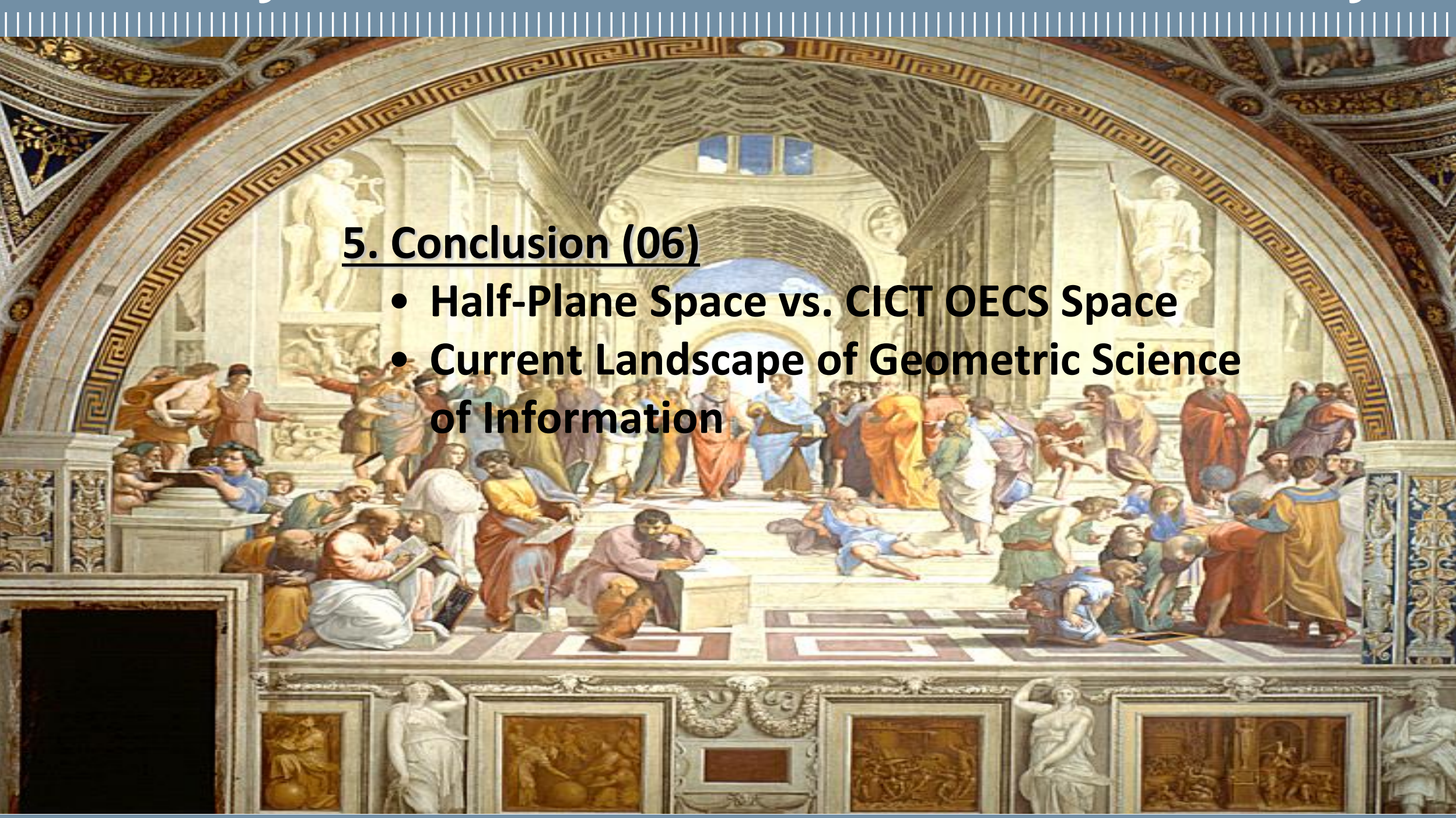

58 Rodolfo A. Fiorini

POLITECNICO MILANO 1863 


\section{The CICT IOU Reference Framework for Stronger AMS System Simulation in Science and Industry}

\section{Siegel Upper-Half Space}

The Poincaré upper-half plane (PUHP) for 2D problems.

The Siegel upper-half space (SUHS) for 3D problems (rotat. symmetry along Y axis).

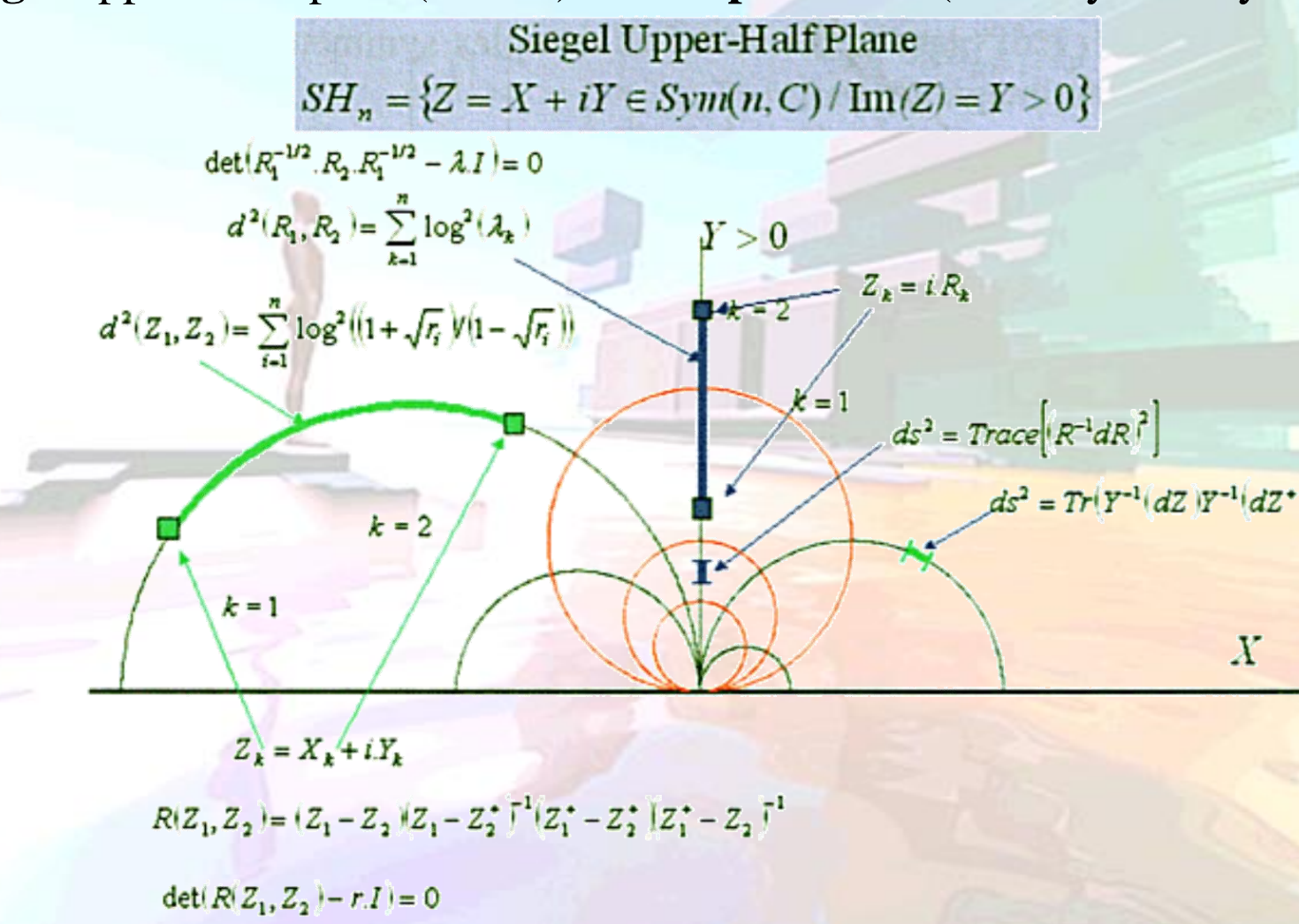

(F. Barbaresco, 2014)

59 Rodolfo A. Fiorini 


\section{The CICT IOU Reference Framework for Stronger AMS System Simulation in Science and Industry}

\section{Half-Plane Space vs. CICT OECS Space}

Two Irreducible Complementary Operative Spaces

\section{Half-Plane Space}

$\square$ Inert matter best operational representation compromise.

A Representation Space endowed with full Flexibility

(mapping complexity to simplicity to give space to Imagination).

- Simplified system dynamics framework (Newtonian Approach).

To model any geometrical space and monitor system dynamics behavior only.

A Spectator can become a system innatural perturbation.
OECS Space

- Livig matter best representation operational compromise.

An Outer Representation Space oneto-one linked to its Inner Representation Space.

- Natural system dynamics framework (Quantum Physics Approach).

- To model projective relativistic geometry and to anticipate emergent system dynamics.

- An Observer can become a system natural co-artifex. 


\section{The CICT IOU Reference Framework for Stronger AMS System Simulation in Science and Industry}

|||||||||||||||||||||||||||||||||||||||||||||||||||||||||||||||||||||||||||||||||||||||||||||||||||||||||||-

\section{Current Landscape of Geometric Science of Information}

Hessian (J.L. Koszul), Homogeneous Convex Cones (E. Vinberg), Homogeneous Symmetric Bounded Domains (E. Cartan, C.L. Siegel), Symplectic (T. von Friedrich, J.M. Souriau), Affine (T. Sasaki, E. Calabi), Information (C. Rao, N. Chentsov). Through Legendre Duality, Contact (V. Arnold) is considered as the odd-dimensional twin of symplectic geometry and could be used to understand Legendre mapping in information geometry.

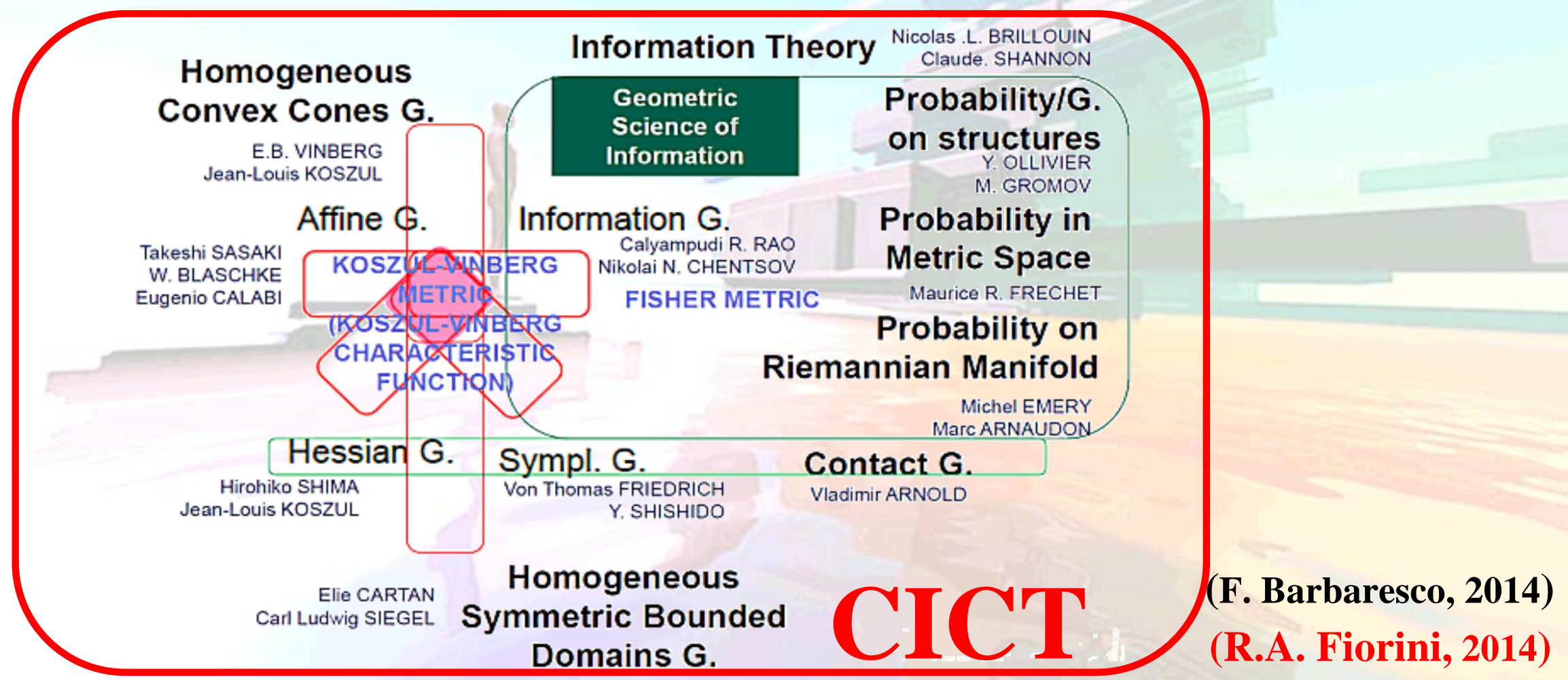


The CICT IOU Reference Framework for Stronger AMS System Simulation in Science and Industry

\section{Immediate Effective Solution to:}

$\square$ circRNA and Bioinformatics

Current Ambiguities;

$\square$ Silent Gene Discovery;

$\square$ Precision Medicine Applications;

$\square$ Complex AMS System Modeling;

口Etc...

62 Rodolfo A. Fiorini 


\section{The CICT IOU Reference Framework for Stronger AMS System Simulation in Science and Industry}

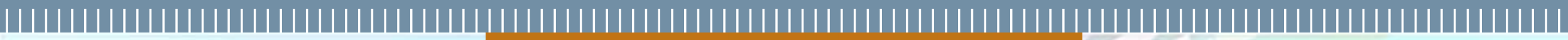
Piero De Giacomo

Rodolfo A. Fiorini

\section{CREATIVITY MIND}

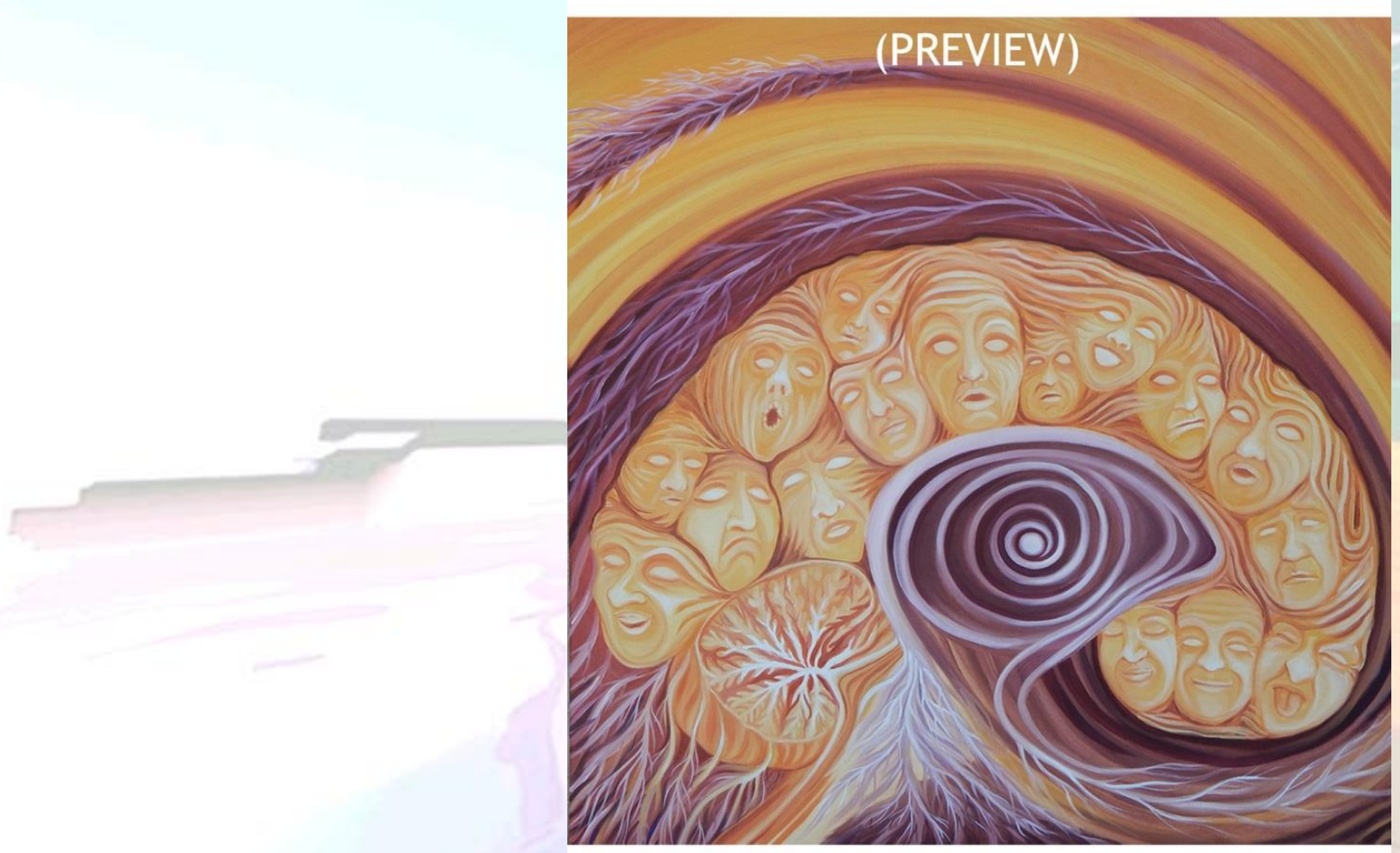

63 Rodolfo A. Fiorini 


\section{The CICT IOU Reference Framework for Stronger AMS System Simulation in Science and Industry}

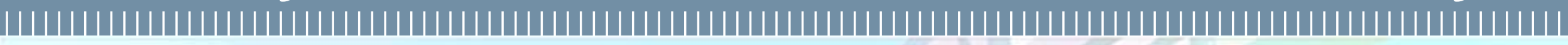

\section{Neuralizer Work In Progress}

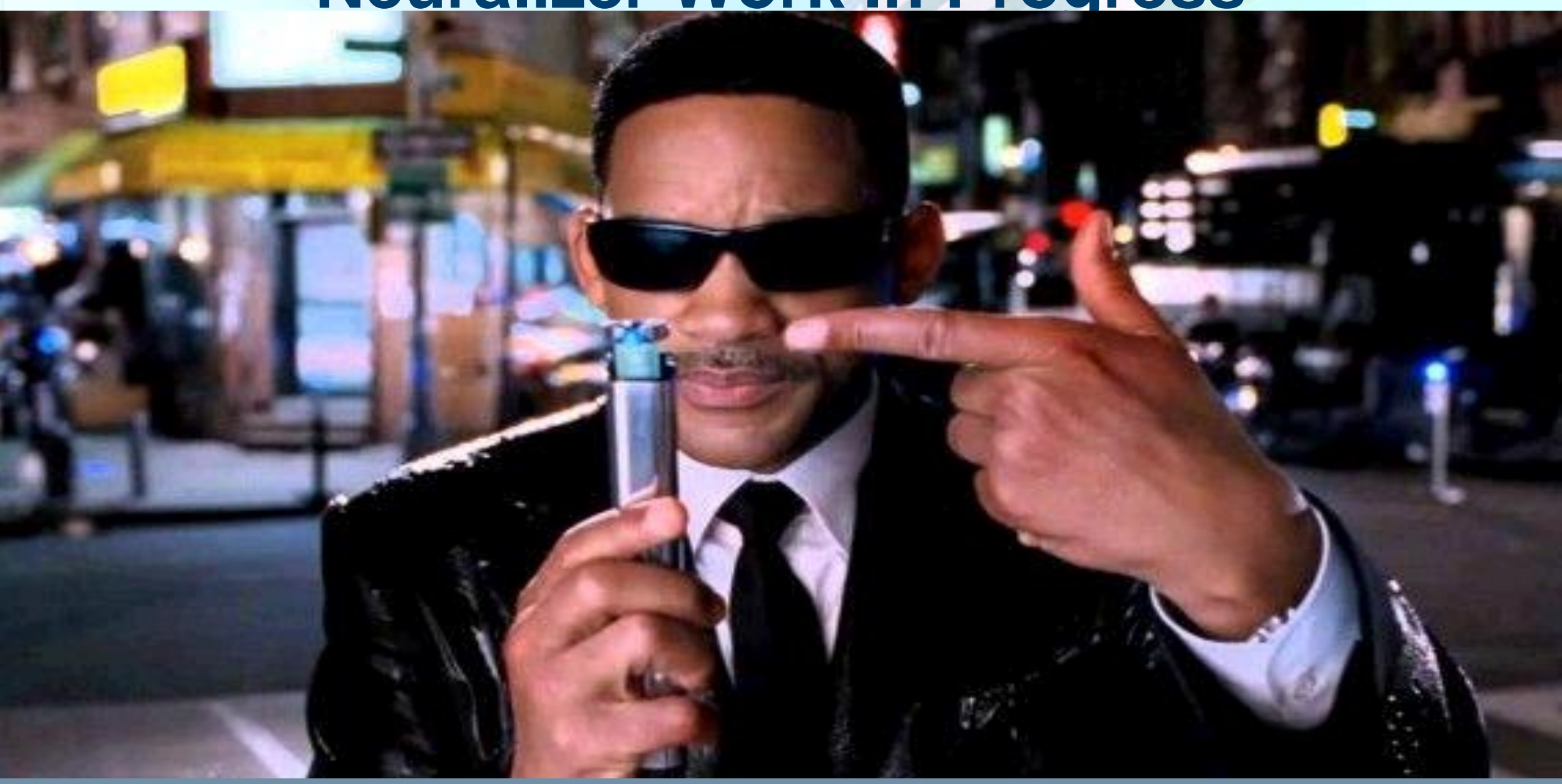

64 Rodolfo A. Fiorini

POLITECNICO MILANO 1863 


\section{The CICT IOU Reference Framework for Stronger AMS System Simulation in Science and Industry}

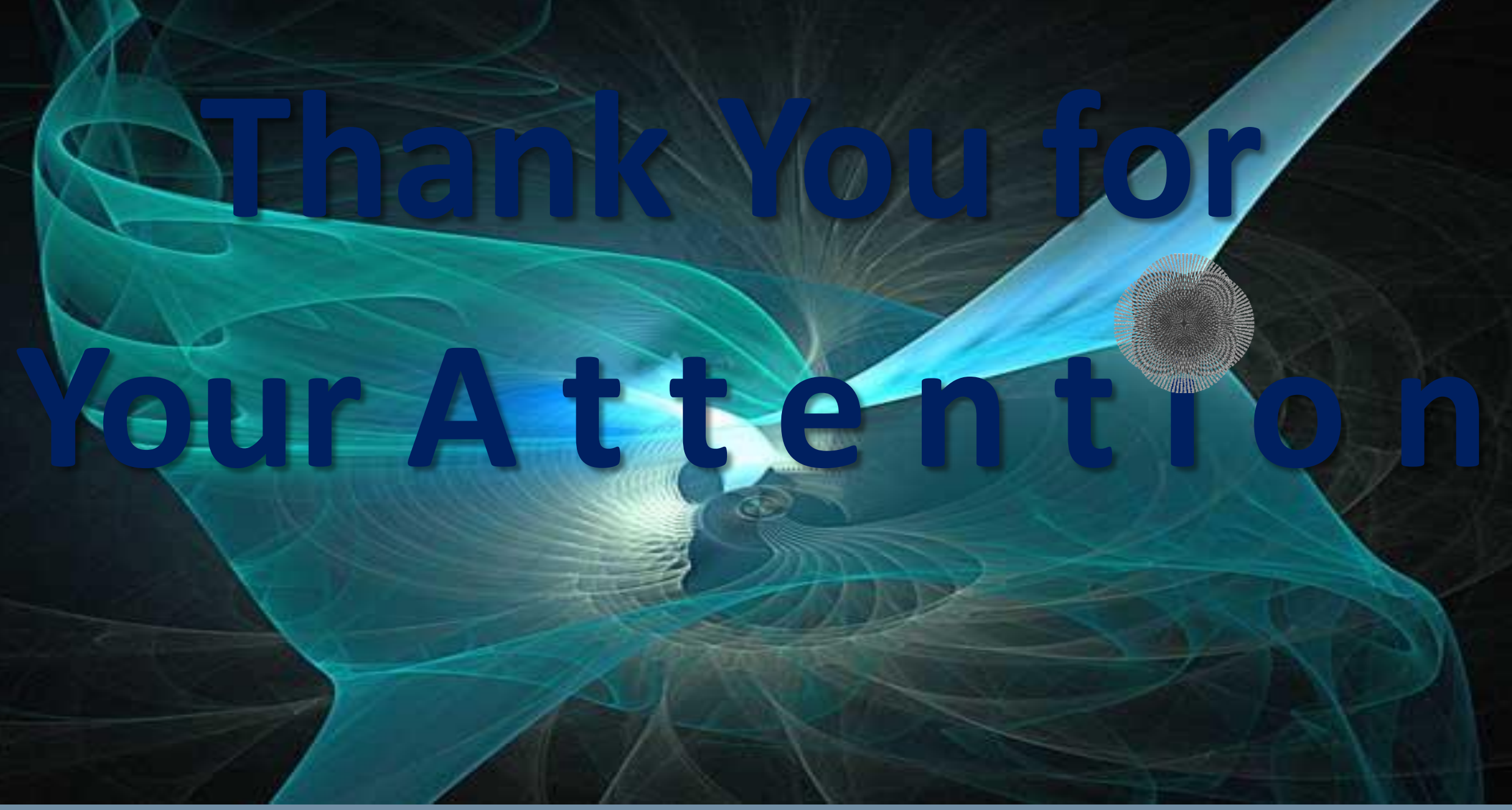

65 Rodolfo A. Fiorini

POLITECNICO MILANO 1863 